\title{
Efeitos do Halotano, Isoflurano e Sevoflurano nas Respostas Cardiovasculares ao Pinçamento Aórtico Infra-Renal. Estudo Experimental em Cães *
}

\section{Effects of Halothane, Isoflurane and Sevoflurane on Cardiovascular Responses to Infrarenal Aortic Cross-Clamping. Experimental Study in Dogs}

\author{
Flora Margarida Barra Bisinotto, $T S A^{1}{ }^{\text {; }}$ José Reinaldo Cerqueira Braz, TSA ${ }^{2}$
}

\section{RESUMO}

Bisinotto FMB, Braz JRC - Efeitos do Halotano, Isoflurano e Sevoflurano nas Respostas Cardiovasculares ao Pinçamento Aórtico Infra-Renal. Estudo Experimental em Cães

JUSTIFICATIVA E OBJETIVOS: O pinçamento infra-renal da aorta abdominal pode produzir alterações hemodinâmicas. O objetivo do estudo foi avaliar os efeitos do halotano, isoflurano e sevoflurano sobre a função cardiovascular, em cães submetidos à pinçamento aórtico infra-renal.

MÉTODO: O estudo aleatório foi realizado em 30 cães, distribuídos em três grupos, de acordo com o anestésico halogenado utilizado durante a anestesia, em concentrações equipotentes de 0,75 CAM: GH $(n=10)$ - halotano a 0,67\%; GI $(n=10)$ - isoflurano a $0,96 \%$; e GS $(n=10)$ - sevoflurano a $1,8 \%$. Em todos os animais foi realizada ligadura infra-renal da aorta, por período de $30 \mathrm{~min}$. Os atributos hemodinâmicos foram estudados nos momentos: C (Controle), A015 e Ao30, respectivamente após 15 e 30 minutos do pinçamento aórtico, e DAo e DAo15, respectivamente, imediatamente e após 15 min do despinçamento aórtico.

RESULTADOS: Durante o pinçamento aórtico houve, em todos os grupos, aumento das pressões arterial média e do átrio direito, e dos índices cardíaco, sistólico e de trabalho sistólico dos ventrículos direito e esquerdo. A pressão da artéria pulmonar aumentou em GI e GS e a pressão pulmonar ocluída em GH e Gl. Após o despinçamento aórtico, houve normalização dos atributos que haviam se elevado, com exceção dos índices cardíaco e sistólico, que continuaram elevados, acompanhados de diminuição do índice de resistência vascular sistêmica. Não houve diferença significante entre os grupos em relação aos atributos estudados, com exceção da freqüência cardíaca que foi

\footnotetext{
${ }^{*}$ Recebido do (Received from) Laboratório Experimental do CET/SBA do Departamento de Anestesiologia da Faculdade de Medicina de Botucatu (FMB) - UNESP, no Programa de Pós-Graduação em Anestesiologia, Doutorado, com equipamentos adquiridos por meio dos Auxílios à Pesquisa $n^{o s}$ 96/3302-0 e 97/09982-6 da FAPESP

1. Pós-Graduanda (Doutorado) do Programa de Pós-Graduação em Anestesiologia da FMB - UNESP. Bolsista do CNPq

2. Professor Titular do CET/SBA do Departamento de Anestesiologia da FMB - UNESP
}

\footnotetext{
Prof. Dr. José Reinaldo Cerqueira Braz

Dept ${ }^{\circ}$ de Anestesiologia da FMB - UNESP

Distrito de Rubião Junior

18618-970 Botucatu, SP
}

Endereço para Correspondência (Correspondence to)

Apresentado (Submitted) em 15 de outubro de 2002

Aceito (Accepted) para publicação em 23 de dezembro de 2002

(C) Sociedade Brasileira de Anestesiologia, 2003 sempre menor em $\mathrm{GH}$, em relação aos demais grupos, durante o pinçamento e despinçamento aórtico.

CONCLUSÕES: No cão, nas condições experimentais empregadas, a inalação do halotano, isoflurano e sevoflurano em concentrações equipotentes (0,75 CAM) não atenua as respostas cardiovasculares ao pinçamento aórtico infra-renal.

Unitermos: ANESTÉSICOS, Vólátil: halotano, isoflurano, sevoflurano; ANIMAL: cão; CIRURGIA, Vascular: pinçamento aórtico infra-renal

\section{SUMMARY}

Bisinotto FMB, Braz JRC - Effects of Halothane, Isoflurane and Sevoflurane on Cardiovascular Responses to Infrarenal Aortic Cross-Clamping. Experimental Study in Dogs

BACKGROUND AND OBJECTIVES: Infrarenal aortic cross-clamping is associated to cardiovascular effects. This study aimed at analyzing the effects of halothane, isoflurane and sevoflurane on cardiovascular function of dogs following infrarenal aortic cross-clamping.

METHODS: Thirty mongrel dogs were randomly divided in three groups, according to equipotent $(0.75 \mathrm{MAC})$ inhaled anesthetic doses: $\mathrm{GH}(n=10)-0.67 \%$ halothane; $\mathrm{GI}(n=10)-0.96 \%$ isoflurane; and GS $(n=10)-1.8 \%$ sevoflurane. All dogs were submitted to infrarenal aortic cross-clamping for 30 minutes. Hemodynamic parameters were measured at control (C), at 15 (Ao15) and 30 (Ao30) minutes of aortic cross-clamping, and immediately (DAo) and 15 (DAo15) minutes after aortic unclamping.

RESULTS: In all groups, infrarenal aortic cross-clamping significantly increased mean blood pressure, right atrial pressure, cardiac index, stroke volume index, left ventricular work index and right ventricular work index. Pulmonary artery pressure significantly increased during cross-clamping in GI and GS groups while pulmonary capillary wedge pressure significantly increased in $\mathrm{GH}$ and $\mathrm{Gl}$ groups. After aortic unclamping all hemodynamic parameters have returned to control levels with the exception of cardiac and stroke volume indices which remained high, followed by systemic vascular resistance index decrease. There have been no significant differences among groups in studied attributes, except for heart rate which was always lower in $\mathrm{GH}$ group as compared to other groups during and after infrarenal aortic cross-clamping.

CONCLUSIONS: In dogs under our experimental conditions, equipotent concentrations ( $0.75 \mathrm{MAC}$ ) of inhalational halothane, isoflurane and sevoflurane have not attenuated cardiovascular responses to infrarenal aortic cross-clamping.

Key Words: ANESTHETICS: Volatile: halothane, isoflurane, sevoflurane; ANIMAL: dog; SURGERY, Vascular: infrarenal aortic cross-clamping 


\section{INTRODUÇÃO}

C erca de $80 \%$ dos aneurismas aórticos surgem abaixo das artérias renais ${ }^{1}$ e a sua correção é um desafio para o cirurgião, assim como para o anestesiologista. Os pacientes são idosos, com função e reserva limitadas de órgãos como coração, pulmões e rins, além de o procedimento impor agressões fisiológicas e dinâmicas expressivas, com grandes alterações hídricas, sangramento importante e eventual comprometimento renal, além das alterações hemodinâmicas secundárias ao pinçamento e despinçamento da aorta ${ }^{2}$.

A taxa de mortalidade, nos casos de cirurgias eletivas de aneurisma abdominal, chegou a ser de $3 \%$, mas ela está diminuindo nos últimos anos, enquanto em casos de ruptura do aneurisma a taxa varia entre $20 \%$ e $50 \%$ dos pacientes ${ }^{2}$. As complicações cardíacas são as maiores causas de morbidade após a cirurgia de reconstrução da aorta ${ }^{2,3}$. A alta incidência de complicações cardíacas resulta, em parte, das alterações fisiopatológicas que ocorrem durante o pinçamento e despinçamento aórticos, do nível do pinçamento, da condição cardíaca basal e do manuseio anestésico durante a cirurgia, determinada pelas ações dos anestésicos no estado do miocárdio e tônus vascular ${ }^{2}$.

Por outro lado, vários fatores têm contribuído para a redução da mortalidade nas cirurgias para correção de aneurisma abdominal, como a melhoria da técnica cirúrgica, a intervenção cirúrgica mais precoce, a melhor seleção dos pacientes, os avanços na monitorização, o surgimento de novas drogas e técnicas anestésicas e o aumento dos cuidados intensivos no pós-operatório ${ }^{2}$.

Os anestésicos podem apresentar papel importante na patogênese das alterações cardíacas ao pinçamento e despinçamento aórticos, por causa de seus efeitos sobre a hemodinâmica cardiovascular e a liberação de hormônios mediadores. Os estudos realizados no homem com halotano e isoflurano durante cirurgia aórtica mostraram comportamento hemodinâmico semelhante durante o pinçamento infra-renal, com diminuição do débito cardíaco e aumento da resistência vascular sistêmica ${ }^{4}$. Por outro lado, os autores verificaram que a profundidade anestésica obtida por diferentes concentrações de halogenados pode alterar a intensidade da resposta hemodinâmica ao pinçamento aórtico, como pode ocorrer com o isoflurano ${ }^{5}$ e desflurano ${ }^{6}$.

Na literatura ao nosso alcance, não se encontrou nenhuma pesquisa relacionada às repercussões hemodinâmicas durante cirurgia da aorta com o emprego de sevoflurano.

O objetivo da pesquisa foi avaliar a função cardiovascular de cães sob anestesia com concentração equipotentes de halotano, isoflurano e sevoflurano, durante pinçamento e despinçamento aórtico infra-renal, com a finalidade de verificar qual dos agentes halogenados associa-se com menores alterações hemodinâmicas.

\section{MÉTODO}

Após aprovação pela Comissão de Ética em Pesquisa Animal local, foram utilizados 30 cães adultos, sem raça definida, de ambos os sexos, com peso entre 18 e $20 \mathrm{~kg}$. Os animais foram distribuídos aleatoriamente em três grupos, com 10 animais em cada grupo, de acordo com o anestésico inalatório halogenado empregado:

GH - Manutenção anestésica com halotano a 0,67\% (0,75 concentração alveolar mínima - CAM);

GI - Manutenção anestésica com isoflurano a 0,96\% (0,75 CAM);

GS - Manutenção anestésica com sevoflurano a 1,8\% (0,75 CAM).

A CAM utilizada dos halogenados no cão foi feita segundo a proposição de Kazama e Ikeda (1988) ${ }^{7}$. Os grupos tiveram duas fases experimentais: na primeira, foi feita indução anestésica, intubação traqueal, instalação de ventilação mecânica, manutenção anestésica com halotano, isoflurano ou sevoflurano, dependendo do grupo estudado, monitorização da ventilação, oxigenação, hemodinâmica cardiovascular e temperatura, seguida de laparotomia mediana para preparação da ligadura aórtica infra-renal da aorta, por um período de 30 minutos, seguido da retirada de ligadura aórtica.

\section{Seqüência Experimental}

Após jejum alimentar de 12 horas, mas com livre acesso à água, os animais, após indução anestésica com propofol $\left(5,5 \mathrm{mg} \cdot \mathrm{kg}^{-1}\right)$ e cloreto de alcurônio $\left(0,2 \mathrm{mg} \cdot \mathrm{kg}^{-1}\right)$, por via venosa, foram colocados em goteira de Claude Bernard, realizando-se a seguir:

1. Intubação orotraqueal e instalação de ventilação controlada a volume, empregando-se o respirador do aparelho de anestesia Excel mod. 210 SE (Ohmeda - EUA) e sistema semifechado com 0,8 L. $\mathrm{min}^{-1}$ de $\mathrm{O}_{2}$ e 1,2 L. $\mathrm{min}^{-1}$ de ar. O volume corrente foi padronizado em $20 \mathrm{ml}^{\mathrm{kg}} \mathrm{kg}^{-1} \mathrm{e}$ a freqüência respiratória em 10 a 15 mov. min $^{-1}$ para manter a pressão expiratória final de $\mathrm{CO}_{2}$ entre 30 e 35 $\mathrm{mmHg}$;

2. Instalação do biomonitor AS3 da Datex Ohmeda (Finlândia) para leitura e registro dos parâmetros ventilatórios, hemodinâmicos, de oxigenação e de temperatura;

3. Instalação do eletrocardiógrafo de três canais (derivação $\left.D_{\|}\right)$, do sensor do termômetro no terço inferior do esôfago, do captador de amostra de gases inspirados e expirados junto à válvula em $\mathrm{Y}$ do circuito respiratório para análise ventilométrica, dos gases e do anestésico halogenado inalados e exalados, e do sensor da saturação periférica da oxihemoglobina $\left(\mathrm{SpO}_{2}\right)$, colocado na língua do animal; 
4. A inalação do agente halogenado foi feita por meio de vaporizador calibrado específico para cada halogenado da Ohmeda (EUA), empregando-se inicialmente 1 CAM do halogenado (halotano a $0,89 \%$, isoflurano a $1,3 \%$ ou sevoflurano $2,4 \%$ ), de acordo com a sua concentração expirada;

5. Dissecção e cateterismo da veia femoral esquerda para infusão contínua da solução de Ringer com lactato (18 $\left.\mathrm{ml} \cdot \mathrm{kg}^{-1} \cdot \mathrm{h}^{-1}\right)$, por meio de bomba de infusão e administração de doses intermitentes do bloqueador neuromuscular alcurônio $\left(0,06 \mathrm{mg} \cdot \mathrm{kg}^{-1}\right)$;

6. Dissecção e cateterismo da artéria femoral esquerda para medida da pressão arterial aórtica e controle do pinçamento e despinçamento aórticos;

7. Dissecção e cateterismo da artéria axilar esquerda para medida da pressão arterial média (PAM) e coleta de sangue para medida do $\mathrm{pH}$ e gases sangüíneos no aparelho Chiron Diagnostics, mod. Rapidlab 865 (Inglaterra);

8. Dissecção e cateterismo da veia jugular externa direita com introdutor 8,5 F e passagem de cateter de Swan-Ganz na artéria pulmonar, para medida do débito cardíaco, por termodiluição, e das pressões;

9. Realização de laparotomia mediana e dissecção infra-renal da aorta. Colocação de fita cardíaca ao redor da aorta, imediatamente após a emergência das artérias renais, para posterior ligadura aórtica. A fita cardíaca foi transpassada em um pequeno tubo plástico de $15 \mathrm{~cm}$. A incisão cirúrgica foi, em seguida, fechada ao redor do tubo plástico;

10. Após o preparo, reduziu-se a CAM dos halogenados, segundo o grupo estudado, de 1 para 0,75 CAM, iniciando-se o período de estabilização hemodinâmica, com duração de 30 minutos;

11. Medida dos atributos e coleta de sangue (momento controle);

12. Injeção de heparina, por via venosa, na dose de 70 Ul. $\mathrm{kg}^{-1}$, e após 3 minutos, realização da ligadura infra-renal da aorta, avançando-se o tubo plástico ao longo da fita cardíaca até que não mais houvesse registro de pressão arterial na artéria femoral;

13. Medida dos atributos e coleta de sangue após 15 (Ao15) e 30 (Ao30) minutos da ligadura aórtica;

14. Retirada da ligadura aórtica e medida dos atributos e coleta de sangue imediatamente (DAo) e após 15 minutos (DAo15) da retirada da ligadura;

15. Término do experimento e sacrifício de animal com excesso de anestésico (pentobarbital sódico).

\section{Atributos Estudados}

Os atributos estudados foram: antropométricos: peso $(\mathrm{kg})$, comprimento $(\mathrm{cm})$, superfície corporal $\left(\mathrm{m}^{2}\right)$; sexo; hemodinâmicos: freqüência cardíaca (FC - bat. $\mathrm{min}^{-1}$ ), pressão arterial média (PAM - $\mathrm{mmHg}$ ), pressão média da artéria pulmonar (PAP - $\mathrm{mmHg}$ ), pressão média do átrio direito (PAD - $\mathrm{mmHg}$ ), pressão da artéria pulmonar ocluída (PAPO- $\mathrm{mmHg}$ ), índices cardíaco (IC - L. $\mathrm{min}^{-1} \cdot \mathrm{m}^{-2}$ ), sistóli- co (IS - ml.bat ${ }^{-1} \cdot \mathrm{m}^{-2}$ ), de resistência vascular pulmonar (IRVP - dina.s. $\mathrm{cm}^{-5} \cdot \mathrm{m}^{-2}$ ), de trabalho sistólico dos ventrículos esquerdo (ITSVE - g.min. $\mathrm{m}^{-2}$ ) e direito (ITSVD g.min. $\mathrm{m}^{-2}$ ); sangüíneos: $\mathrm{pH}$ arterial $(\mathrm{pHa})$ e pressão parcial de dióxido de carbono arterial $\left(\mathrm{PaCO}_{2}-\mathrm{mmHg}\right)$; e temperatura: esofagiana $\left(\mathrm{T}_{\text {Esof }}-{ }^{\circ} \mathrm{C}\right)$.

\section{Análise Estatística}

Os atributos estudados foram submetidos à Análise de Perfil ${ }^{8}$. Para as variáveis antropométricas, utilizou-se ANOVA e para a distribuição do sexo, utilizou-se o teste Qui-quadrado. As estatísticas foram consideradas significantes quando $p<$ 0,05 .

\section{RESULTADOS}

Os grupos mostraram-se homogêneos em relação aos valores antropométricos e a distribuição do sexo (Tabela I).

Tabela I - Atributos Antropométricos e Distribuição do Sexo nos Grupos

\begin{tabular}{lcccc}
\hline Grupos & $\begin{array}{c}\text { Peso * } \\
(\mathrm{kg})\end{array}$ & $\begin{array}{c}\text { Comprimento * } \\
(\mathrm{cm})\end{array}$ & $\begin{array}{c}\text { Superfície Corporal * } \\
\left(\mathrm{m}^{2}\right)\end{array}$ & $\mathrm{M} / \mathrm{F}$ \\
\hline $\mathrm{GH}$ & $18,3 \pm 1,6$ & $101,2 \pm 7,8$ & $0,702 \pm 0,067$ & $7 / 3$ \\
$\mathrm{GI}$ & $19,9 \pm 2,0$ & $103,0 \pm 5,6$ & $0,731 \pm 0,043$ & $6 / 4$ \\
$\mathrm{GS}$ & $19,7 \pm 2,1$ & $104,1 \pm 5,2$ & $0,736 \pm 0,061$ & $8 / 2$ \\
\hline
\end{tabular}

Não houve diferença significante entre os grupos $(p>0,05)$ * Valores expressos em Média \pm DP

O único atributo hemodinâmico que apresentou alteração significante entre os grupos foi a FC, que no grupo $\mathrm{GH}$ apresentou menores valores em Ao15 em relação aos grupos GI e GS, e em Ao30 em relação ao grupo GI (Figura 1). Nos grupos, durante o pinçamento aórtico, houve aumento da PAM em todos os grupos (Ao15 e Ao30), acompanhado por aumento significante da PAD em todos os grupos (Figura 2), da PAP nos grupos GI e GS (Figura 3), da PAPO nos grupos GH e GI (Figura 4), do IC e do IS em todos os grupos (Figuras 5 e 6), e do ITSVD e ITSVE em todos os grupos (Figuras 7 e 8). Já os valores da IRVS diminuíram significantemente, em todos os grupos, após o despinçamento aórtico (Figura 9), enquanto os valores do IRVP diminuíram significantemente durante o pinçamento aórtico em GS (Figura 10). Após o despinçamento aórtico, houve diminuição significante em todos os grupos, em relação ao pinçamento aórtico, dos valores da PAM (Figura 2), da PAD (Figura 3); já os valores do ITSVE diminuíram significantemente nos grupos GI e GS (Figuras 10 e 11), enquanto os valores da IC e IS permaneceram significantemente elevados em todos os grupos (Figuras 6 e 7, respectivamente). 


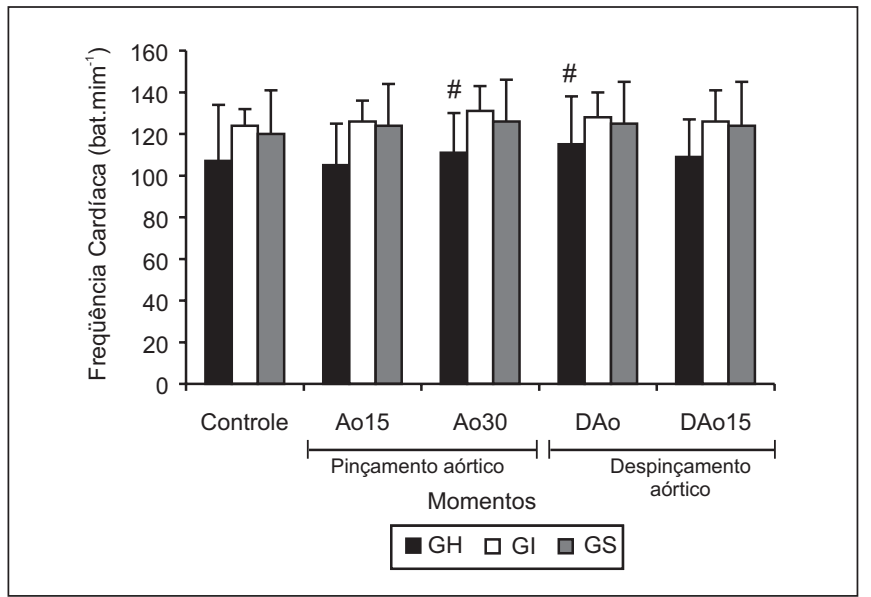

Figura 1 - Freqüência Cardíaca (Média \pm DP)

$\# p<0,05$ :

Ao30: $\mathrm{GH}<(\mathrm{GI}=\mathrm{GS})$

DAo: $\mathrm{GH}<\mathrm{Gl}$

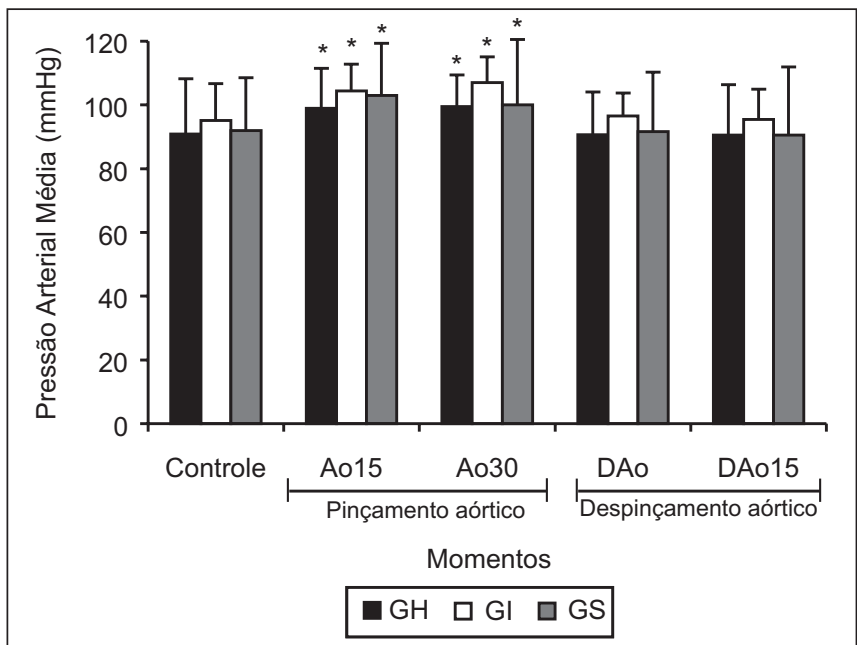

Figura 2 - Pressão Arterial Média (Média \pm DP)

${ }^{*} p<0,05$ :

$\mathrm{GH}: \mathrm{C}<(\mathrm{Ao} 15=\mathrm{Ao} 30)>(\mathrm{DAo}=\mathrm{DA0} 15)$

Gl: $\mathrm{C}<(\mathrm{Ao15}=\mathrm{Ao} 30)>(\mathrm{DAo}=\mathrm{DA0} 15)$

GS: $C<($ Ao15 $=$ Ao30 $)>(D A o=D A 015)$

Os valores do $\mathrm{pHa}$ apresentaram diminuição significante após o pinçamento aórtico em GH e GI e após a despinçamento aórtico em GS (Tabela II), enquanto os valores da $\mathrm{PaCO}_{2}$ não se alteraram significantemente entre os grupos (Tabela II). Já os valores da $\mathrm{T}_{\text {Esof }}$ diminuíram significantemente, em todos os grupos, ao longo do experimento (Tabela II).

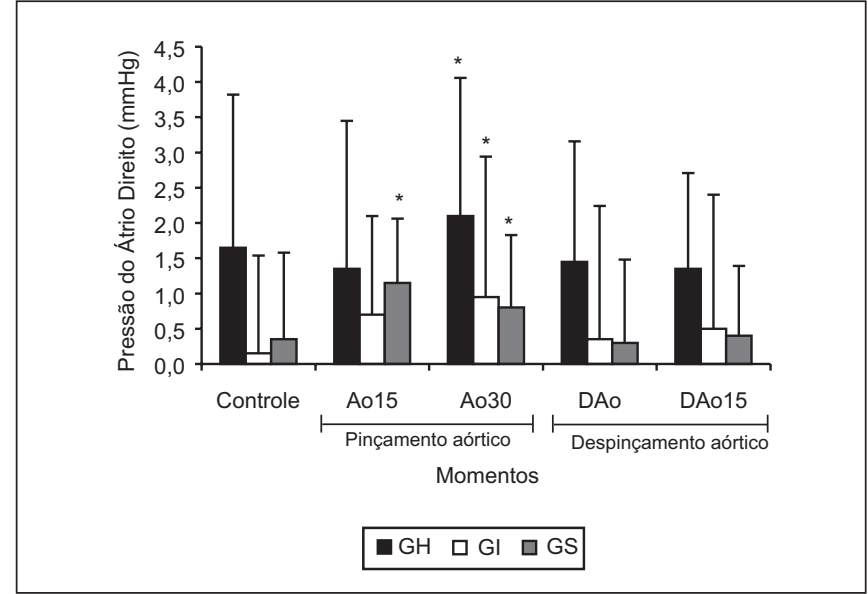

Figura 3 - Pressão do Átrio Direito (Média \pm DP) $* p<0,05$ :

$\mathrm{GH}:(\mathrm{C}=\mathrm{Ao15})<\mathrm{Ao30}>(\mathrm{DAo}=\mathrm{DA0} 15)$

Gl: $(C=A 015)<A 030>(D A 0=D A 015)$

GS: $\mathrm{C}<($ Ao15 $=$ Ao30 $)>(D A o=D A 015)$

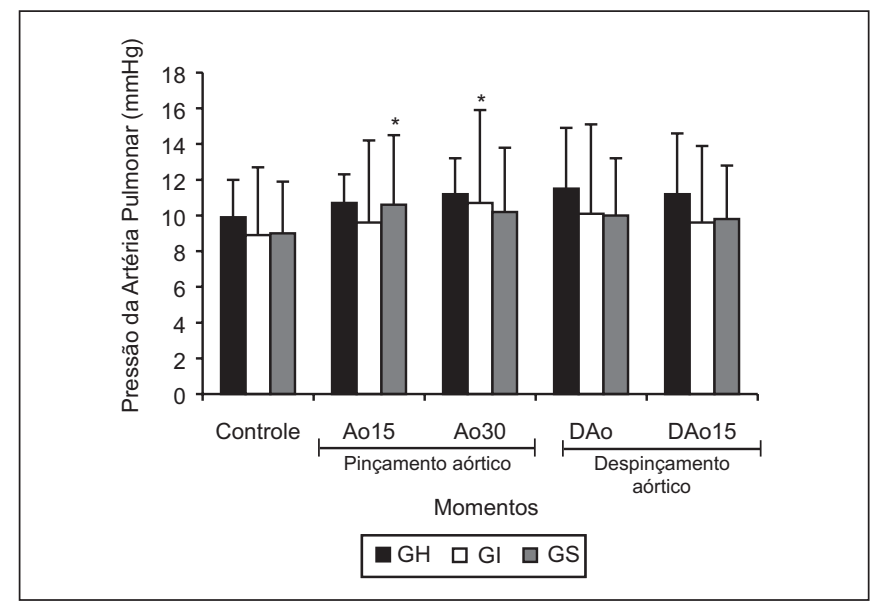

Figura 4 - Pressão da Artéria Pulmonar (Média \pm DP) ${ }^{*} p<0,05$ :

GI: C $<$ Ao30

GS: C $<$ Ao15

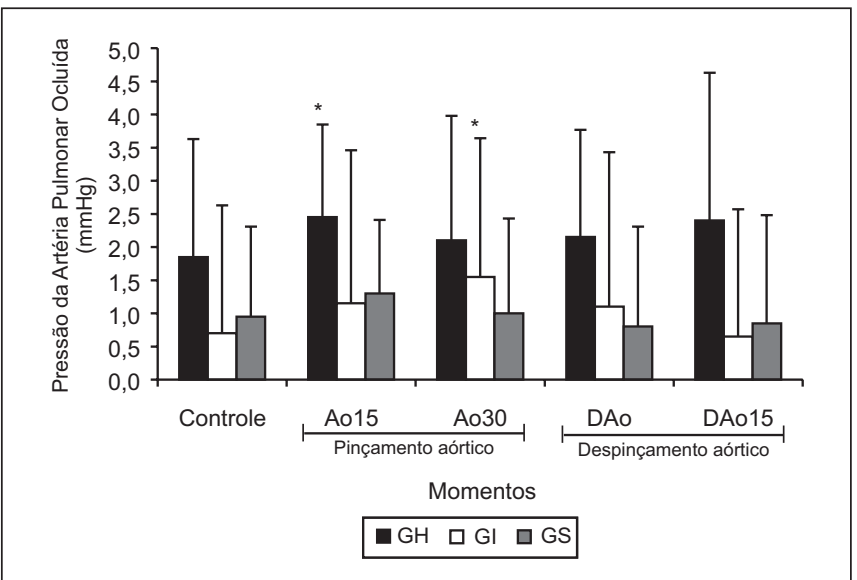

Figura 5 - Pressão da Artéria Pulmonar Ocluída (Média \pm DP) * $p<0,05$ :

$\mathrm{GH}: \mathrm{C}<\mathrm{Ao} 15$

GI: C < A030 > DA015

Revista Brasileira de Anestesiologia Vol. 53, No 4, Julho - Agosto, 2003 


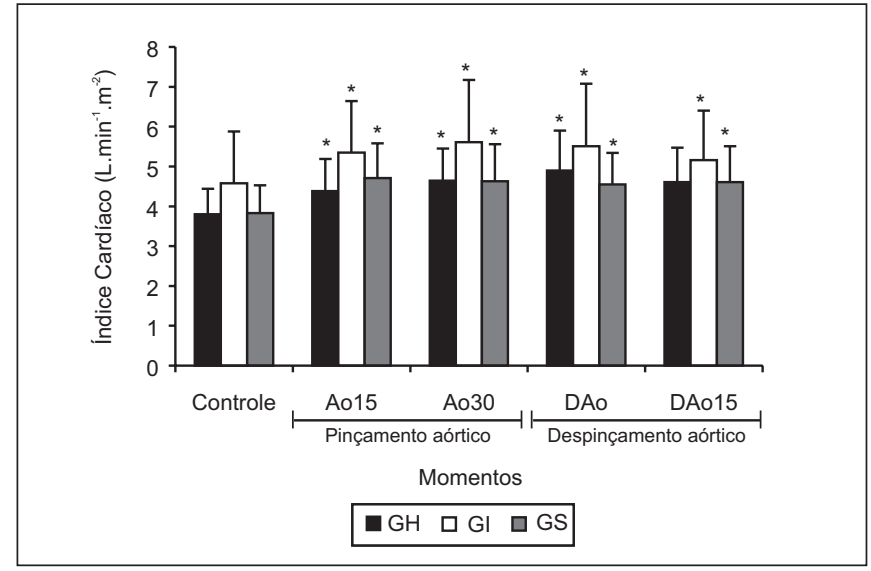

Figura 6 - Índice Cardíaco (Média $\pm \mathrm{DP}$ ) ${ }^{*} p<0,05$

$\mathrm{GH}: \mathrm{C}<($ Ao15 $=$ Ao30 $=\mathrm{DAo})$

GI: $\mathrm{C}<($ Ao15 $=$ Ao30 $=$ DAo $=$ DAo15 $)$

GS: $C<($ Ao15 $=$ Ao30 $=$ DAo $=$ DAo15 $)$

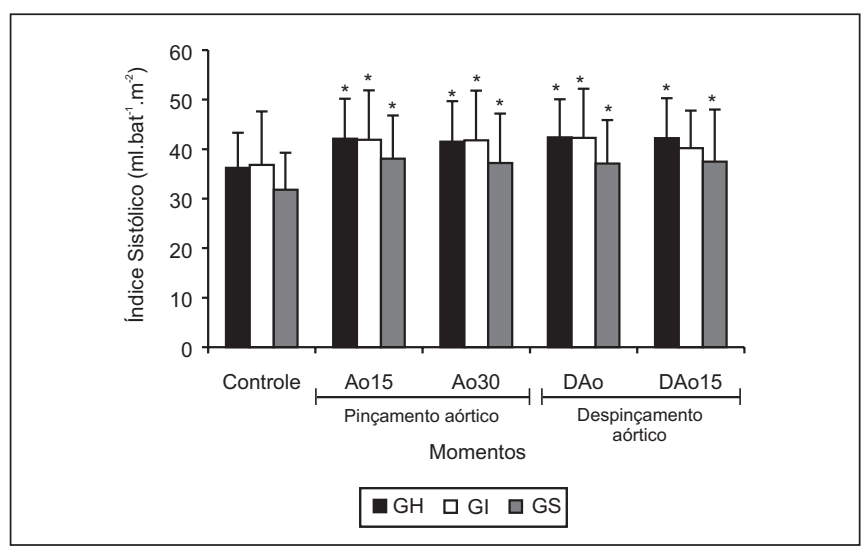

Figura 7 - Índice Sistólico (Média $\pm \mathrm{DP}$ )

* $p<0,05$ :

$\mathrm{GH}: \mathrm{C}<(\mathrm{Ao15}=\mathrm{Ao} 30=\mathrm{DAo}=\mathrm{DA015})$

GI: $\mathrm{C}<($ Ao15 = Ao30 = DAo $)$

GS: $C<($ Ao15 $=$ Ao30 $=$ DAo $=$ DAo15 $)$

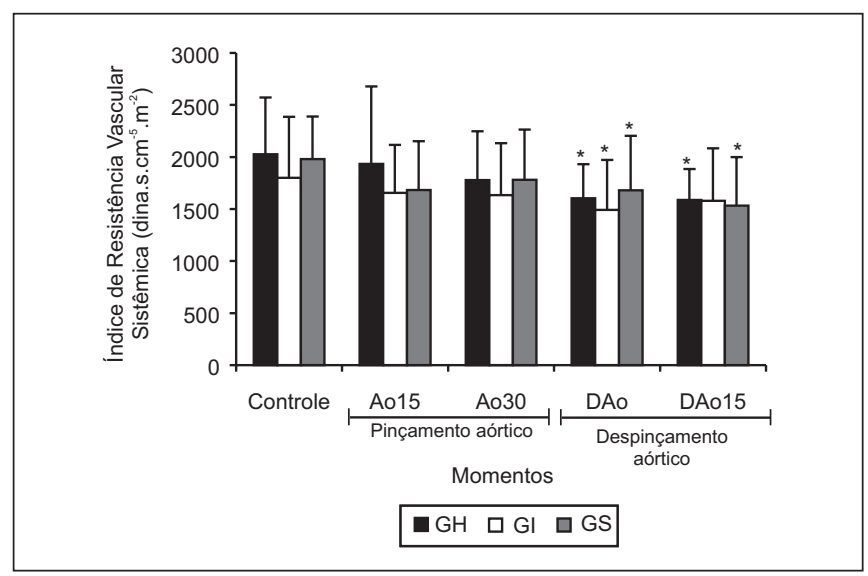

Figura 8 - Índice de Resistência Vascular Sistêmica (Média \pm DP)

$$
\begin{aligned}
& { }^{*} p<0,05: \\
& G H:(C=A o 15)>(D A o=D A 015) \\
& G I: C>D A o
\end{aligned}
$$$$
\text { GS: } \mathrm{C}>(\mathrm{DAo}=\mathrm{DAo} 15)
$$

Revista Brasileira de Anestesiologia Vol. 53, № 4, Julho - Agosto, 2003

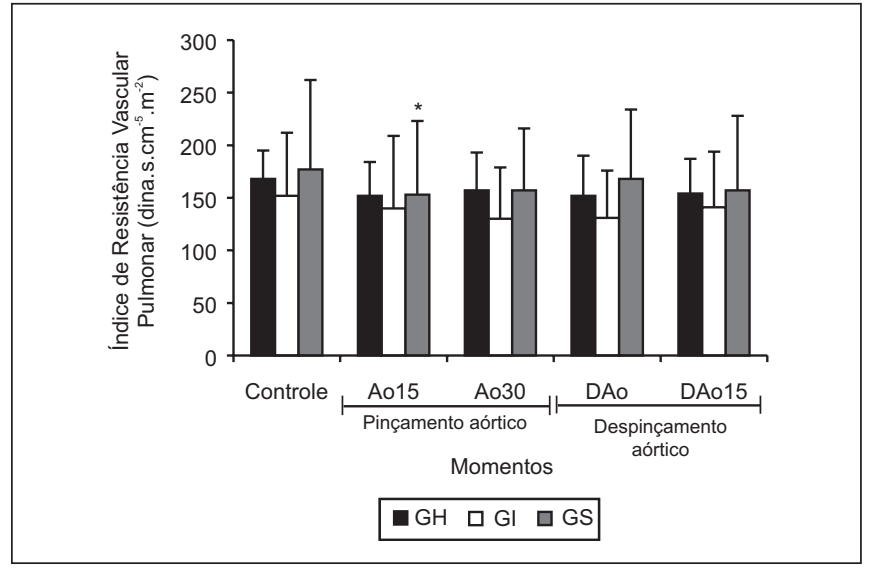

Figura 9 - Índice de Resistência Vascular Pulmonar (Média \pm DP) ${ }^{*} p<0,05$ :

GS: C > Ao15

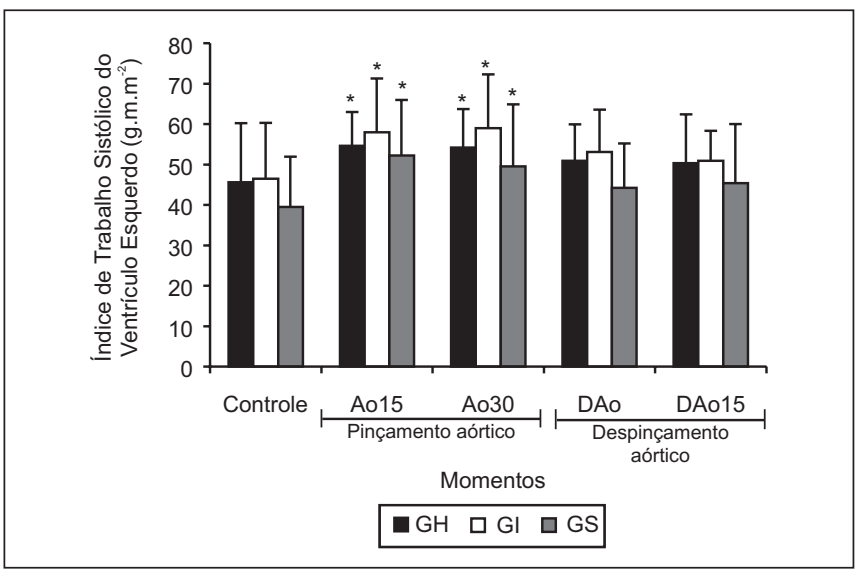

Figura 10 - Índice de Trabalho Sistólico do Ventrículo Esquerdo (Média $\pm \mathrm{DP}$ )

${ }^{*} p<0,05$ :

$\mathrm{GH}: \mathrm{C}<($ Ao15 = Ao30)

GI: $C<(A 015=A 030)>$ DAo15

GS: $C<($ Ao15 = Ao30 $)>$ DAo15

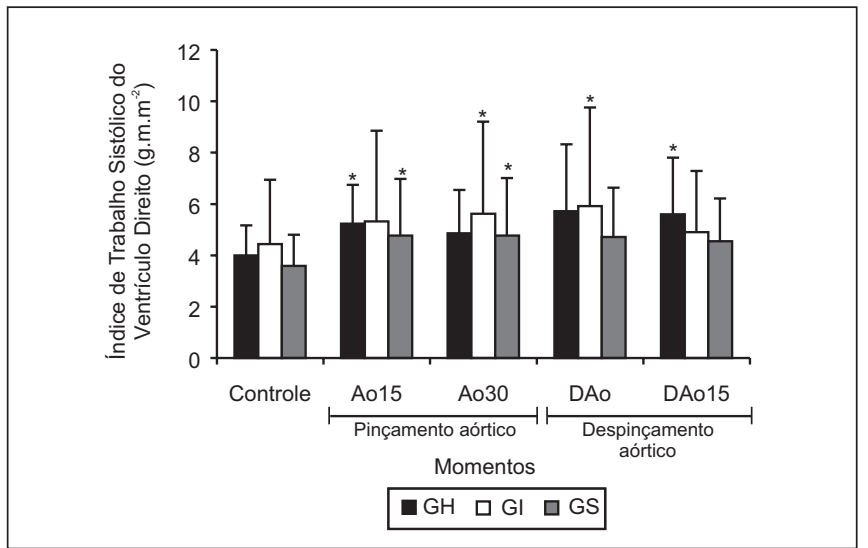

Figura 11 - Índice de Trabalho Sistólico do Ventrículo Direito (Média $\pm \mathrm{DP}$ )

$\mathrm{GH}: \mathrm{C}<\mathrm{Ao} 15<(\mathrm{DAo}=\mathrm{DA0} 15)$

$\mathrm{GI}: \mathrm{C}<(\mathrm{Ao} 30=\mathrm{DAO})$

GS: $C<($ Ao15 = Ao30 $)$ 
Tabela II - pH Arterial (pHa), Pressão Parcial de Dióxido de Carbono Arterial $\left(\mathrm{PaCO}_{2}\right)$ e Temperatura Esofágica ( $\left.\mathrm{T}_{\mathrm{Esof}}\right)$, (Média \pm DP)

\begin{tabular}{|c|c|c|c|c|c|c|}
\hline & & & & Momentos & & \\
\hline Atributos & Grupos & Controle & Ao15 & Ao30 & DAo & DAo15 \\
\hline \multirow[t]{3}{*}{$\mathrm{pHa}$} & $\mathrm{GH}$ & $7,35 \pm 0,07$ & $7,33 \pm 0,06$ & $7,31 \pm 0,05^{*}$ & $7,31 \pm 0,06^{*}$ & $7,29 \pm 0,06^{*}$ \\
\hline & $\mathrm{GI}$ & $7,37 \pm 0,06$ & $7,33 \pm 0,05^{*}$ & $7,33 \pm 0,05^{*}$ & $7,31 \pm 0,04^{*}$ & $7,32 \pm 0,05^{*}$ \\
\hline & GS & $7,33 \pm 0,06$ & $7,31 \pm 0,06$ & $7,31 \pm 0,06$ & $7,28 \pm 0,06^{*}$ & $7,28 \pm 0,05^{*}$ \\
\hline \multirow[t]{3}{*}{$\mathrm{PaCO}_{2}(\mathrm{mmHg})$} & $\mathrm{GH}$ & $30 \pm 4$ & $30 \pm 6$ & $32 \pm 6$ & $32 \pm 6$ & $33 \pm 6$ \\
\hline & $\mathrm{Gl}$ & $31 \pm 5$ & $32 \pm 5$ & $30 \pm 6$ & $33 \pm 5$ & $32 \pm 3$ \\
\hline & GS & $34 \pm 4$ & $35 \pm 5$ & $35 \pm 4$ & $35 \pm 4$ & $36 \pm 4$ \\
\hline \multirow[t]{3}{*}{$\mathrm{T}_{\text {Esof }}\left({ }^{\circ} \mathrm{C}\right)$} & $\mathrm{GH}$ & $37,0 \pm 0,8$ & $36,7 \pm 0,8^{*}$ & $36,7 \pm 0,8^{*}$ & $36,6 \pm 0,9^{*}$ & $36,4 \pm 0,8^{*}$ \\
\hline & $\mathrm{GI}$ & $37,2 \pm 0,8$ & $36,9 \pm 0,8^{*}$ & $36,9 \pm 0,9^{*}$ & $36,7 \pm 0,9^{*}$ & $36,6 \pm 0,9^{*}$ \\
\hline & GS & $37,3 \pm 0,6$ & $36,8 \pm 1,0^{*}$ & $36,8 \pm 0,6^{*}$ & $36,7 \pm 0,6^{*}$ & $36,5 \pm 0,7^{*}$ \\
\hline
\end{tabular}

* $p<0,05$ em relação ao momento controle do mesmo grupo

\section{DISCUSSÃO}

O estudo confirmou a presença de alterações hemodinâmicas após o pinçamento e despinçamento aórticos, mesmo quando realizados em níveis mais inferiores da aorta, como o pinçamento infra-renal. Essas alterações, que ocorreram sem diferença significante nos três grupos, com exceção da freqüência cardíaca que foi significantemente menor no grupo halotano em relação aos demais grupos, foram caracterizadas por aumento, em pequena proporção (média de 10\% a $13 \%$ ), da PAM, que é sempre a resposta mais observada durante o pinçamento aórtico, acompanhado por aumento das pressões de enchimento, como PAD, PAP e PAPO, e de aumento do IC, IS, ITSVE e ITSVD, com diminuição significante dos seus valores após o despinçamento aórtico.

Vários autores observaram aumento das pressões de enchimento durante o pinçamento aórtico ${ }^{9,10}$, enquanto outros autores não observaram essas alterações ${ }^{5}$. Por outro lado, muitos autores atribuem, como causa principal do aumento da PAM durante o pinçamento aórtico, o aumento súbito da impedância do fluxo aórtico, com aumento da pós-carga ${ }^{11}$, acompanhado, muitas vezes, por diminuição do $I C^{9}$. Na presente pesquisa, o IRVS não se alterou significantemente em nenhum dos grupos estudados durante o pinçamento aórtico, enquanto o IRVP diminuiu significantemente, no grupo sevoflurano, durante o pinçamento aórtico.

Assim, a resposta hemodinâmica que ocorreu durante o pinçamento aórtico na presente pesquisa pode ser explicada, ao menos em parte, pela ocorrência de redistribuição do volume sangüíneo. Assim, Quintin e col. (1990) ${ }^{12}$ observaram, no homem, recolhimento venoso passivo distal ao pinçamento aórtico, com liberação de catecolaminas (adrenalina e noradrenalina) e de angiotensina. A liberação dessas substâncias determinou vasoconstrição tanto proximal quanto distal ao pinçamento, com diminuição do sistema de capacitância e deslocamento do volume sangüíneo proximal ao pinçamento, com grande interferência no retorno venoso, ou seja, na pré-carga, na dependência do nível do pinçamen472 to aórtico ser supra ou infra-celíaco. Na primeira situação, o retorno venoso aumentou sempre, porque o deslocamento do volume sangüíneo ocorreu em direção aos músculos proximais ao pinçamento e aos pulmões e cérebro. Já no infra-celíaco, houve deslocamento do volume sangüíneo em direção aos órgãos esplâncnicos ou para outros tecidos proximais ao pinçamento. Caso o tônus venoso esplâncnico esteja diminuído, haverá diminuição do retorno venoso ${ }^{13}$. Como conseqüência, a distribuição de sangue entre a vasculatura esplâncnica e a não esplâncnica é que irá determinar as alterações da pré-carga.

Além do nível do pinçamento aórtico, principal fator determinante das alterações hemodinâmicas ${ }^{9}$, outros fatores também podem ser importantes na determinação das respostas ao pinçamento aórtico, como as alterações do volume sangüíneo ou do tônus vascular esplâncnico determinados pela hidratação; o estado anestésico ou as ações de drogas, os quais podem alterar tanto a redistribuição do volume sangüíneo como o retorno venoso; a função ventricular; a presença e significância da doença isquêmica do miocárdio; e o tipo de doença da aorta, aneurismática ou doença oclusiva ${ }^{2}$.

Quanto aos efeitos dos anestésicos halogenados em pacientes com ausência de doenças cardiovasculares e submetidos à cirurgia eletiva, verificou-se que o halotano, em doses eqüipotentes, é mais depressor miocárdico do que o isoflurano ou o sevoflurano ${ }^{14,15}$, enquanto o sevoflurano e, principalmente, o isoflurano exercem maior efeito vasodilatador sobre a resistência vascular sistêmica do que o halotano ${ }^{16} \mathrm{e}$ mantêm a FC mais elevada do que o halotano ${ }^{17}$.

Na pesquisa, o fato de se ter empregado doses eqüipotentes e não elevadas $(0,75 \mathrm{CAM})$ de cada um dos anestésicos halogenados certamente foi decisivo nos resultados hemodinâmicos obtidos. Ressalte-se que a FC foi o único atributo que apresentou diferença significante entre os grupos, com menores valores no grupo halotano em relação aos demais grupos, nos momentos do pinçamento aórtico.

A comparação dos efeitos hemodinâmicos do isoflurano e sevoflurano em pacientes com doença miocárdica isquêmi-

Revista Brasileira de Anestesiologia Vol. 53, No 4, Julho - Agosto, 2003 
ca e hipertensão arterial mostra também comportamento semelhante ${ }^{18}$. Colson e col. ${ }^{19}$ (1992) verificaram em pacientes submetidos à cirurgia para reconstrução aórtica infra-renal, sob anestesia com halotano a $0,64 \%$ ou isoflurano a $0,77 \%$, que não houve diferença significante dos variáveis hemodinâmicas entre os grupos, no período pré-pinçamento, como FC, PAM, PAPO e IRVS. Durante o pinçamento aórtico infra-renal, o comportamento hemodinâmico também foi o mesmo, com diminuição do IC e aumento do IRVS, sem que ocorressem alterações entre os grupos da FC, PAM ePAPO. Os resultados obtidos em cães demonstram também similaridade das respostas hemodinâmicas com o isoflurano e sevoflurano, com manutenção do IC, às custas de aumento da FC e diminuição do IRVS. Já o halotano, por não alterar significantemente a FC e por ser depressor miocárdico mais potente do que os demais halogenados, não mantém o IC como aumento da profundidade anestésica (2 CAM) ${ }^{20}$.

Seeman-Lodding e col. $(1996)^{5}$, em modelo experimental de pinçamento infra-renal da aorta em porcos sob anestesia de base com cetamina e cloralose, verificaram durante o pinçamento, aumento da PAM ( $17 \% \pm 4 \%)$ e do IRVS $(27 \% \pm 7 \%)$, sem que ocorressem alterações significantes do IC, FC, PAD, PAP, PAPO e IRVP. No mesmo estudo, os autores introduziram um segundo grupo, que recebeu isoflurano primeiramente a $0,7 \%$ e após a $1,4 \%$, antes de cada pinçamento, que determinou diminuição dose-dependente da PAM e IRVS, enquanto a FC e IC diminuíram somente com a maior concentração do halogenado, mas sem que houvesse alterações significantes da PAD, PAP e PAPO. Durante o pinçamento aórtico, o isoflurano a $0,7 \%$ não alterou a resposta hemodinâmica, mas a $1,4 \%$ diminuiu o IRVS e a PAM. Com o emprego do isoflurano nas duas concentrações, houve diminuição da PAM, em níveis inferiores aos do grupo controle, mas durante o pinçamento, somente a concentração mais elevada determinou níveis de PAM inferiores aos do momento controle do grupo controle.

No modelo experimental por nós utilizado, os mecanismos que determinaram aumento da PAM durante o pinçamento, como vimos anteriormente, foram diferentes dos do modelo experimental descrito anteriormente. Mesmo assim, empregando o isoflurano em concentração intermediária à utilizada pelos autores anteriores, houve, durante o pinçamento aórtico, o mesmo comportamento da PAM observado no grupo que recebeu isoflurano a $0,7 \%$, ou seja, não houve impedimento para que houvesse pequeno aumento da PAM.

Utilizando modelo experimental em porcos semelhante ao descrito anteriormente, Sundeman e col. (1996) ${ }^{6}$ observaram que o desflurano a $4,9 \%$ não alterou significantemente a intensidade da resposta hemodinâmica ao pinçamento aórtico infra-renal. No entanto, o desflurano a 9,8\% diminuiu a PAM e o IRVS durante o pinçamento.

Após o despinçamento aórtico, houve diminuição da maioria dos atributos hemodinâmicos que havia se elevado durante o pinçamento aórtico, acompanhado de diminuição significante, em todos os grupos, do IRVS, o que favoreceu a manutenção do IC e IS em valores acima dos do controle nos grupos.

Revista Brasileira de Anestesiologia

Vol. 53, N 4, Julho - Agosto, 2003
Diferentes respostas hemodinâmicas podem ser observadas após o despinçamento aórtico, de acordo com o local do pinçamento aórtico e de sua duração, e do estado hemodinâmico do paciente, ou seja, do estado da volemia e do nível da hemoglobina. Mas há, sempre, diminuição do IRVS e da PAM, com gênese multifatorial ${ }^{2}$. A resposta inicial, observada já após alguns segundos da retirada do pinçamento, sugere um reflexo ou fenômeno mecânico, ou seja, o fluxo sangüíneo aórtico aumenta, preenchendo o espaço criado pela diminuição do tônus vasomotor, provavelmente resultante da hipóxia tecidual, do acúmulo de componentes vasodepressores produzidos pelos tecidos isquêmicos e da resposta miogênica ${ }^{2,21}$. O seqüestro de sangue nas veias distais à oclusão e o aumento do fluxo sangüíneo nos membros inferiores são, aparentemente, os responsáveis pela diminuição do retorno venoso e, conseqüentemente, da diminuição da PAD, do IC e da PAM após o despinçamento aórtico. Por isso, há a necessidade de reposição volêmica adequada para prevenir essas alterações.

Em estudo experimental no porco sob anestesia de base com cetamina e cloralose, observou-se, após cinco minutos do despinçamento aórtico infra-renal, que todos os tributos hemodinâmicos que se alteraram durante o pinçamento aórtico retornaram aos níveis do controle, com exceção da PAM $(+10 \%)$ e do IRVS (+12\%). Nesse mesmo estudo, no grupo que recebeu desflurano a $4,9 \%$, todos as variáveis hemodinâmicas retornaram aos níveis de controle após o despinçamento aórtico, enquanto que com o desflurano a $9,8 \%$, houve diminuição da PAM $(-7 \%)^{6}$.

Em experimentos clínicos em que se realizou pinçamento aórtico infra-renal, seguido de despinçamento, houve diminuição do IRVS, da PAM e do retorno venoso ${ }^{10,19}$. No entanto, oIS e o IC podem não se alterar ${ }^{22}$, aumentar ${ }^{23}$ ou diminuir $^{24}$, na dependência do enchimento do ventrículo esquerdo.

Algumas limitações do estudo experimental em relação à cirurgia aórtica realizada no homem devem ser destacadas. Assim, o tempo de pinçamento aórtico foi relativamente pequeno e certamente insuficiente para determinar alterações hemodinâmicas tempo-dependentes. O estudo foi desenvolvido em cães saudáveis com capacidade adaptativa aparentemente normal da espécie às diferentes situações hemodinâmicas, enquanto no homem a cirurgia aórtica é realizada, geralmente, em pacientes idosos, com algum grau de disfunção miocárdica, com doença oclusiva coronariana, hipertensão arterial, doença pulmonar obstrutiva crônica, disfunção renal e diabetes mellitus, portanto com reserva limitada dos órgãos. Também deve-se considerar que no experimento, diferentemente do que acontece durante a cirurgia aórtica no homem, o sangramento foi pequeno e realizou-se boa expansão volêmica, contribuindo para a manutenção das pressões de enchimento e mesmo atenuando as alterações hemodinâmicas decorrentes do despinçamento aórtico.

Embora tenha sido tomado cuidado com a temperatura ambiente que permaneceu entre $24 \mathrm{e} 25^{\circ} \mathrm{C}$, e tenha-se recoberto os cães com campos cirúrgicos, houve diminuição da temperatura esofágica, em todos os grupos, ocasionando hipo- 
termia de grau leve a moderado nos cães, pois a temperatura central na espécie é de 38 a $39^{\circ} \mathrm{C}^{25}$. Certamente o fato de ter sido utilizado solução glicosada a $5 \%$ gelado como indicador para medida do débito cardíaco, pode também ter colaborado para o desenvolvimento de hipotermia nos animais, pois cada resultado consistiu de três medidas consecutivas, utilizando-se, assim, $30 \mathrm{ml}$ do indicador em cada momento estudado, o que totalizou a infusão de $150 \mathrm{ml}$ de solução glicosada gelada durante a experimentação. Apesar de sua instalação, a intensidade de hipotermia certamente não foi suficiente para alterar os resultados obtidos na pesquisa.

Durante e após o pinçamento aórtico, houve, em todos os grupos, a ocorrência de acidose metabólica, que provavelmente se deveu à isquemia dos tecidos abaixo do pinçamento.

Em conclusão, no cão nas condições experimentais empregadas, a inalação de halotano, isoflurano e sevoflurano em concentrações eqüipotentes $(0,75 \mathrm{CAM})$ não determina diferença significante do comportamento dos principais atributos hemodinâmicos durante o pinçamento aórtico, caracterizado por pequeno aumento das pressões de enchimento e de pressão arterial média. A única exceção é o comportamento da freqüência cardíaca, que apresenta menores valores com o halotano durante o pinçamento aórtico. A utilização de baixas concentrações dos halogenados certamente influenciou os resultados obtidos.

\section{Effects of Halothane, Isoflurane and Sevoflurane on Cardiovascular Responses to Infrarenal Aortic Cross-Clamping. Experimental Study in Dogs}

Flora Margarida Barra Bisinotto TSA, M.D., José Reinaldo Cerqueira Braz TSA, M.D.

\section{INTRODUCTION}

Approximately $80 \%$ of aortic aneurysms are located below renal arteries ${ }^{1}$ and their correction is a challenge both for surgeons and anesthesiologists. These are elderly patients with limited function and reserve of organs such as heart, lungs and kidneys, and the procedure imposes expressive physiological and dynamic aggressions with severe hydric changes, major bleeding and possible renal involvement, in addition to hemodynamic changes secondary to aortic cross-clamping and unclamping ${ }^{2}$.

Mortality rate for abdominal aneurysm elective surgeries has reached $3 \%$ but is recently decreasing, while aneurysm rupture rates vary from $20 \%$ to $50 \%{ }^{2}$. Heart complications after aortic correction surgeries are major morbidity causes ${ }^{2,3}$. This high incidence of heart complications is partly a consequence of pathophysiological changes caused by aortic cross-clamping and unclamping, the clamping level, heart baseline conditions and anesthetic handling during surgery, determined by anesthetic actions on myocardial status and vascular tone ${ }^{2}$.

On the other hand, several factors have contributed to decrease mortality in abdominal aneurysm correction surgeries, such as improved surgical technique, earlier surgical intervention, better patients selection, monitoring advances, new drugs and anesthetic techniques and improved postoperative intensive care ${ }^{2}$.

Anesthetics may play an important role in the pathogenesis of cardiac changes during aortic cross-clamping and unclamping, due to their effects on cardiovascular hemodynamics and to mediator hormones release. Human studies with halothane and isoflurane during aortic surgeries have shown similar hemodynamic behavior during infrarenal cross-clamping, with cardiac output decrease and systemic vascular resistance increase ${ }^{4}$. Conversely, some authors have observed that the anesthetic depth obtained by different halogenate concentrations may change hemodynamic responses to aortic cross-clamping, as it is the case with isoflurane ${ }^{5}$ and desflurane ${ }^{6}$.

We have not found in the available literature any study related to hemodynamic repercussions during aortic surgery with sevoflurane.

This study aimed at evaluating cardiovascular function of dogs anesthetized with equipotent concentrations of halothane, isoflurane and sevoflurane during infra-renal cross-clamping and unclamping, aiming at observing which halogenate agent was associated to lower hemodynamic changes.

\section{METHODS}

After the local Animal Research Ethics Committee approval, 30 adult mongrel dogs of both genders, weighing 18 to $20 \mathrm{~kg}$ were used. Animals were randomly distributed in three groups of 10 animals, according to the halogenate inhalational anesthetic drug used.

$\mathrm{GH}$ - Anesthetic maintenance with $0.67 \%$ halothane $(0.75$ minimum alveolar concentration - MAC);

$\mathrm{GI}$ - Anesthetic maintenance with $0.96 \%$ isoflurane $(0.75$ MAC);

GS - Anesthetic maintenance with $1.8 \%$ sevoflurane $(0.75$ $M A C)$.

Halogenate MAC used in dogs was according to Kazama and Ikeda's proposition (1988) ${ }^{7}$. Groups had two experimental stages: the first consisted of anesthetic induction, tracheal intubation, mechanical ventilation, anesthetic maintenance with halothane, isoflurane or sevoflurane, depending on the group, monitoring of ventilation, oxygenation, cardiovascular hemodynamics and temperature, followed by medial laparotomy to prepare infrarenal aortic cross-clamping for 30 minutes, followed by aortic unclamping. 


\section{Experimental Sequence}

After 12 hours fast but with free access to water, animals were placed in Claude-Bernard device and anesthetic induction with intravenous propofol $\left(5.5 \mathrm{mg} \cdot \mathrm{kg}^{-1}\right)$ and alcuronium $(0.2$ $\mathrm{mg} \cdot \mathrm{kg}^{-1}$ ), being then performed:

1. Tracheal intubation and volume-controlled ventilation using the ventilator of the anesthesia machine Excel mod 210 SE (Ohmeda - USA) and a semi-closed system with $0.8 \mathrm{~L}$. $\mathrm{min}^{-1} \mathrm{O}_{2}$ and $1.2 \mathrm{~L}$. $\mathrm{min}^{-1}$ air. Tidal volume was standardized at $20 \mathrm{ml}^{\mathrm{kg}} \mathrm{kg}^{-1}$ and respiratory rate at 10 to 15 mov. min $^{-1}$ to maintain $\mathrm{CO}_{2}$ end expiratory pressure between 30 and $35 \mathrm{mmHg}$;

2. Installation of Datex Ohmeda (Finland) biomonitor AS3 for reading and recording ventilatory, hemodynamic, oxygenation and temperature parameters;

3. Installation of three-channel electrocardiographer $\left(D_{\|}\right.$ lead), of thermometer sensor in the lower esophageal third, of the inspired and expired gases sampler close to the $Y$ valve of the respiratory circuit for ventilation, gases and inhaled and exhaled halogenate anesthetics analysis, and of the peripheral oxygen saturation sensor $\left(\mathrm{SpO}_{2}\right)$, placed on animal's tongue;

4. Halogenate agent inhalation was performed through Ohmeda gaged vaporizers specific for each halogenate (USA) using initially 1 MAC $(0.89 \%$ halothane, $1.3 \%$ isoflurane or $2.4 \%$ sevoflurane) according to expired concentration;

5. Left femoral vein dissection and catheterization for lactated Ringer's solution continuous infusion $\left(18 \mathrm{ml} \cdot \mathrm{kg}^{-1} \cdot \mathrm{h}^{-1}\right)$ through infusion pump and administration of intermittent alcuronium doses $\left(0.06 \mathrm{mg} \cdot \mathrm{kg}^{-1}\right)$;

6 . Left femoral artery dissection and catheterization for aortic blood pressure and aortic cross-clamping and unclamping control;

7. Left axillary artery dissection and catheterization for mean blood pressure (MBP) control and blood collection to measure $\mathrm{pH}$ and blood gases in the Chiron Diagnostics mod. Rapidlab 865 (UK) device;

8. Right external jugular vein dissection and catheterization with $8.5 \mathrm{~F}$ introducer and Swan-Ganz catheter insertion in the pulmonary artery to measure pressures and cardiac output by thermodilution;

9. Medial laparotomy and infra-renal aortic dissection. Placement of cardiac tape around the aorta, immediately after renal arteries emergence, for posterior aortic ligation. Cardiac tape was passed in a small $15 \mathrm{~cm}$ plastic tube. Surgical incision was then closed around the plastic tube;

10. After preparation, halogenate MAC was decreased, according to the studied group, from 1 to $0.75 \mathrm{MAC}$, starting a 30-minute period of hemodynamic stabilization;

11. Attributes measurement and blood collection (control moment);

12. Intravenous $70 \mathrm{Ul} . \mathrm{kg}^{-1}$ heparin injection and infrarenal aortic ligation 3 minutes, later, advancing the plastic tube along the cardiac tape until there was no longer blood pressure recorded in the femoral artery;

13. Attributes measurement and blood collection 15 (Ao15) and 30 (Ao30) minutes after aortic cross-clamping;

14. Aortic clamping removal and attributes measurement and blood collection immediately (Dao) and 15 minutes. (Dao15) after unclamping;

15. End of experiment and animals' sacrifice with an overdose of sodium pentobarbital.

\section{Studied Attributes}

Studied attributes were: anthropometric: weight $(\mathrm{kg})$, length $(\mathrm{cm})$, body surface $\left(\mathrm{m}^{2}\right)$; gender; hemodynamic: heart rate (HR - bat. $\mathrm{min}^{-1}$ ), mean blood pressure (MBP - mmHg), mean pulmonary artery pressure (PAP - $\mathrm{mmHg}$ ), mean right atrium pressure (RAP - mmHg), pulmonary capillary wedge pressure (PCWP - mmHg), cardiac index $\left(\mathrm{Cl}-\mathrm{L} \cdot \mathrm{min}^{-1} \cdot \mathrm{m}^{-2}\right)$, systolic index $\left(\mathrm{SI}-\mathrm{ml}\right.$.beat $\left.{ }^{-1} \cdot \mathrm{m}^{-2}\right)$, pulmonary vascular resistance index (PVRI - dina.s. $\mathrm{cm}^{-5} \cdot \mathrm{m}^{-2}$ ), left ventricular systolic work (LVSW - g. $\mathrm{min}^{-1} \cdot \mathrm{m}^{-2}$ ), right ventricular systolic work (RVSW - g. $\mathrm{min}^{-1} \cdot \mathrm{m}^{-2}$ ); blood: arterial $\mathrm{pH}(\mathrm{pHa}$ ) and partial arterial carbon dioxide pressure $\left(\mathrm{PaCO}_{2}-\mathrm{mmHg}\right)$; temperature: esophageal $\left(\mathrm{T}_{\mathrm{Esoph}}-{ }^{\circ} \mathrm{C}\right)$.

\section{Statistical Analysis}

Attributes were submitted to Profile Analysis ${ }^{8}$. ANOVA was used for anthropometric data and Chi-square test for gender distribution. Significance level was $p<0.05$.

\section{RESULTS}

Groups were homogeneous in anthropometric data and gender distribution (Table I).

Table I - Anthropometric Data and Gender Distribution within Groups

\begin{tabular}{lcccc}
\hline Groups & $\begin{array}{c}\text { Weight * } \\
(\mathrm{kg})\end{array}$ & $\begin{array}{c}\text { Length * } \\
(\mathrm{cm})\end{array}$ & $\begin{array}{c}\text { Body Surface * } \\
\left(\mathrm{m}^{2}\right)\end{array}$ & M/F \\
\hline $\mathrm{GH}$ & $18.3 \pm 1.6$ & $101.2 \pm 7.8$ & $0.702 \pm 0.067$ & $7 / 3$ \\
$\mathrm{GI}$ & $19.9 \pm 2.0$ & $103.0 \pm 5.6$ & $0.731 \pm 0.043$ & $6 / 4$ \\
GS & $19.7 \pm 2.1$ & $104.1 \pm 5.2$ & $0.736 \pm 0.061$ & $8 / 2$ \\
\hline
\end{tabular}

There were no significant differences among groups $(p>0.05)$

* Values expressed in Mean \pm SD

The single hemodynamic attribute with significant changes among groups was $\mathrm{HR}$ which was lower in $\mathrm{GH}$ at Ao15 as compared to groups $\mathrm{Gl}$ and $\mathrm{GS}$, and at $\mathrm{Ao} 30$ as compared to group GI (Figure 1). MBP increased in all groups during aortic cross-clamping (Ao15 and Ao30), followed by significant increase in DBP for all groups (Figure 2), in PAP in groups GI and GS (Figure 3), in PCWP in groups GH and GI (Figure 4), in 


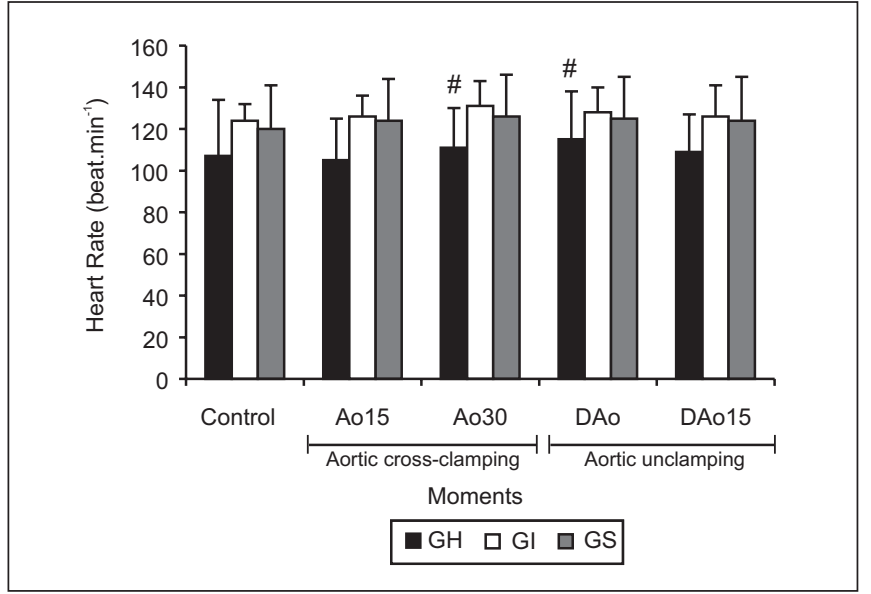

Figure 1 - Heart Rate (Mean $\pm S D$ )

$\# p<0.05$ :

Ao30: $\mathrm{GH}<(\mathrm{GI}=\mathrm{GS})$

DAo: $\mathrm{GH}<\mathrm{Gl}$

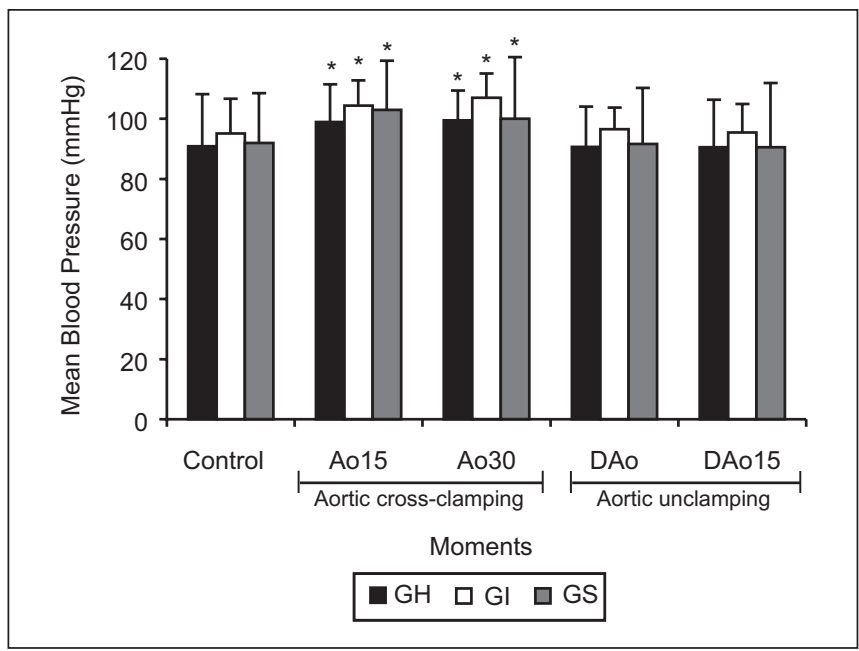

Figure 2 - Mean Blood Pressure (Mean \pm SD)

$$
p<0.05 \text { : }
$$

$\mathrm{GH}: \mathrm{C}<(\mathrm{Ao} 15=\mathrm{Ao} 30)>(\mathrm{DAo}=\mathrm{DA0} 15)$

GI: $C<($ Ao15 $=$ Ao30 $)>(D A 0=D A 015)$

GS: $\mathrm{C}<(\mathrm{Ao} 15=\mathrm{Ao} 30)>(\mathrm{DAO}=\mathrm{DA0} 15)$

$\mathrm{Cl}$ and $\mathrm{SI}$ in all groups (Figures 5 and 6), and in LVSW and RVSW in all groups (Figures 7 and 8 ). SVRI has significantly decreased in all groups after aortic unclamping (Figure 9), while PVRI has significantly decreased during aortic cross-clamping in GS (Figure 10). After aortic unclamping there has been a significant MBP (Figure 2) and DBP (Figure 3 ) decrease in all groups as compared to aortic cross-clamping; LVSW has significantly decreased in groups GI and GS (Figures 10 and 11), while $\mathrm{Cl}$ and $\mathrm{SI}$ have remained significantly high in all groups (Figures 6 and 7, respectively). There has been a significant $\mathrm{pH}$ decrease after aortic cross-clamping in $\mathrm{GH}$ and $\mathrm{Gl}$ and after aortic unclamping in GS (Table II), while $\mathrm{PaCO}_{2}$ has not significantly changed among groups (Table II). There has been a significant $T_{\text {Esoph }}$ decrease in all groups throughout the experiment (Table II).

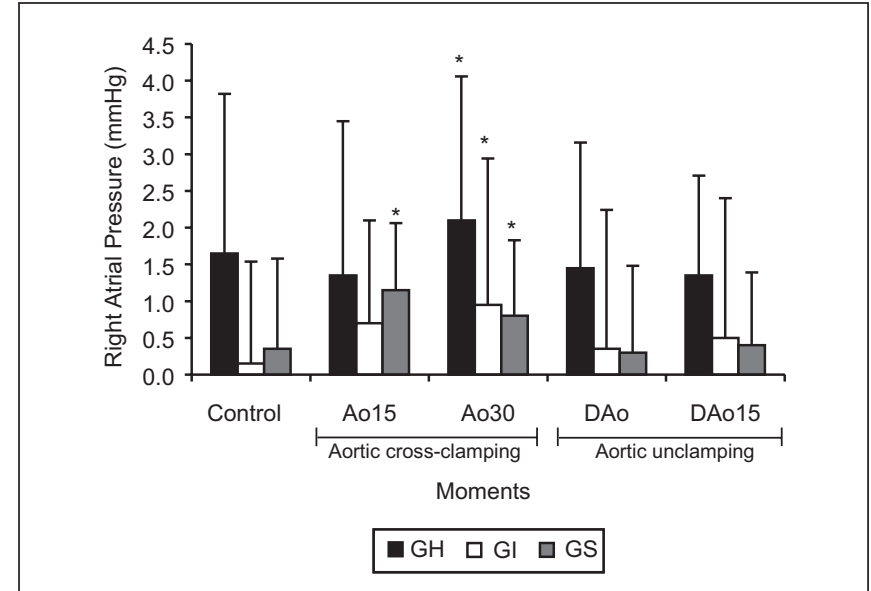

Figure 3 - Right Atrial Pressure (Mean $\pm \mathrm{SD}$ ) ${ }^{*} p<0.05$ :

$\mathrm{GH}:(\mathrm{C}=\mathrm{Ao15})<\mathrm{Ao30}>(\mathrm{DAo}=\mathrm{DA0} 15)$

Gl: $(\mathrm{C}=\mathrm{Ao15})<\mathrm{Ao30}>(\mathrm{DAo}=\mathrm{DA} 015)$

GS: $C<(A 015=A 030)>(D A o=D A 015)$

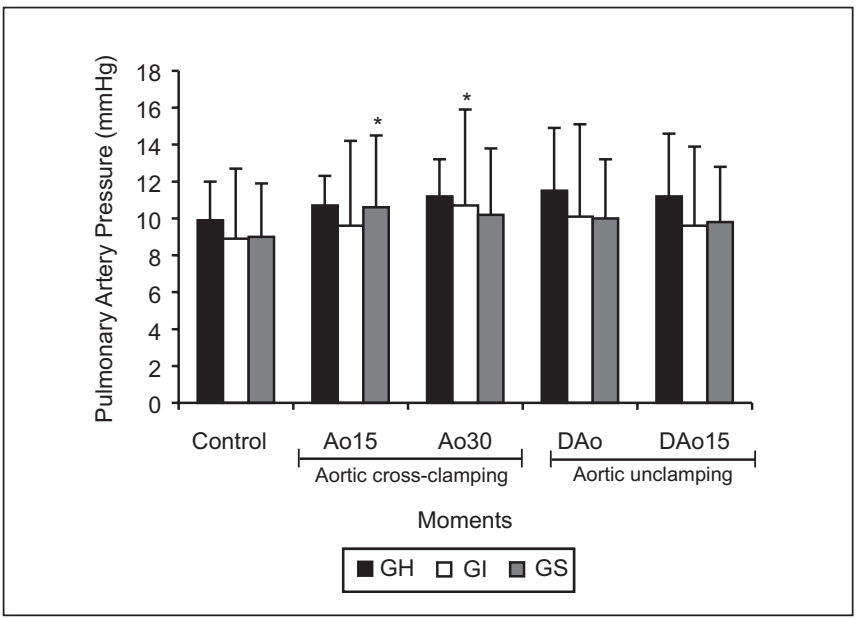

Figure 4 - Pulmonary Artery Pressure (Mean \pm SD) ${ }^{*} p<0.05$ :

GI: C < Ao30

GS: C < Ao15

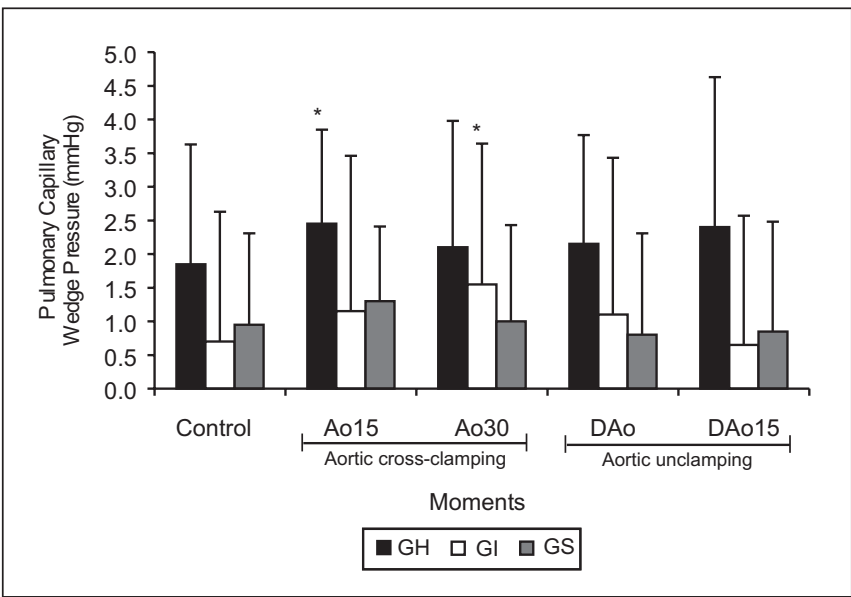

Figure 5 - Pulmonary Capillary Wedge Pressure (Mean \pm SD) ${ }^{*} p<0.05$ :

$\mathrm{GH}: \mathrm{C}<\mathrm{Ao} 15$

GI: C < Ao30 > DAo15 


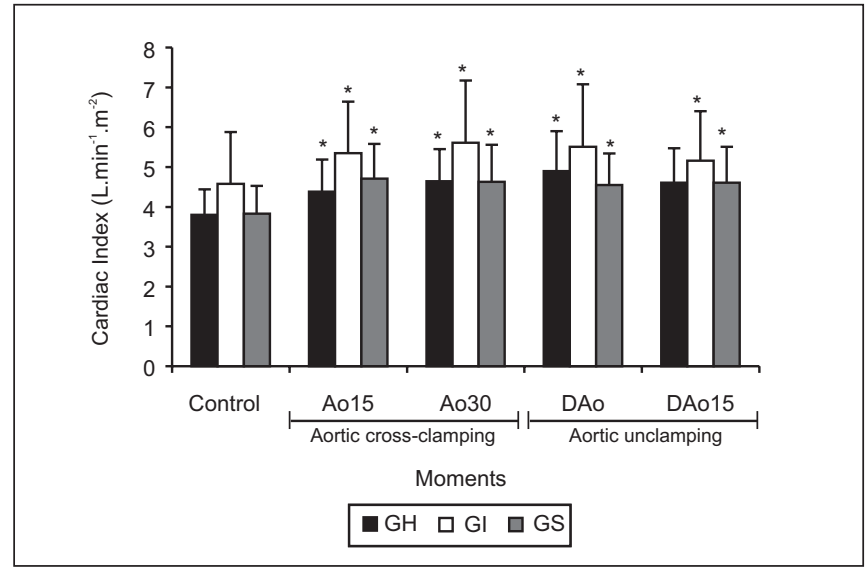

Figure 6 - Cardiac Index (Mean $\pm \mathrm{SD}$ )

${ }^{*} \mathrm{p}<0.05$

$\mathrm{GH}: \mathrm{C}<(\mathrm{Ao} 15=\mathrm{Ao} 30=\mathrm{DAo})$

$\mathrm{Gl}: \mathrm{C}<(\mathrm{Ao15}=\mathrm{AO} 30=\mathrm{DAo}=\mathrm{DA0} 15)$

GS: $C<($ Ao15 $=$ Ao30 $=$ DAo $=$ DAo15 $)$

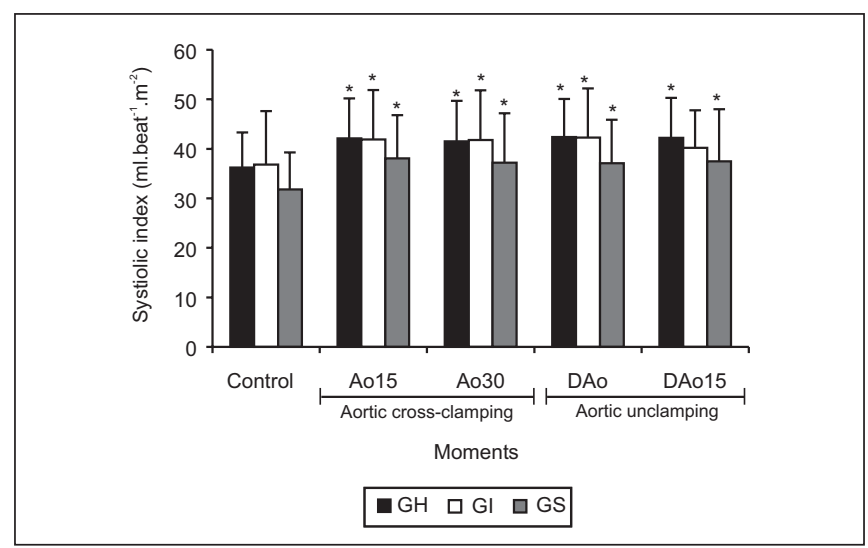

Figure 7 - Systolic Index (Mean \pm SD)

${ }^{*} p<0.05$

$\mathrm{GH}: \mathrm{C}<(\mathrm{Ao} 15=\mathrm{Ao} 30=\mathrm{DAo}=\mathrm{DA0} 15)$

Gl: $C<(A 015=$ Ao30 $=$ DAo $)$

GS: $C<($ Ao15 $=$ Ao30 $=$ DAo $=$ DA015 $)$

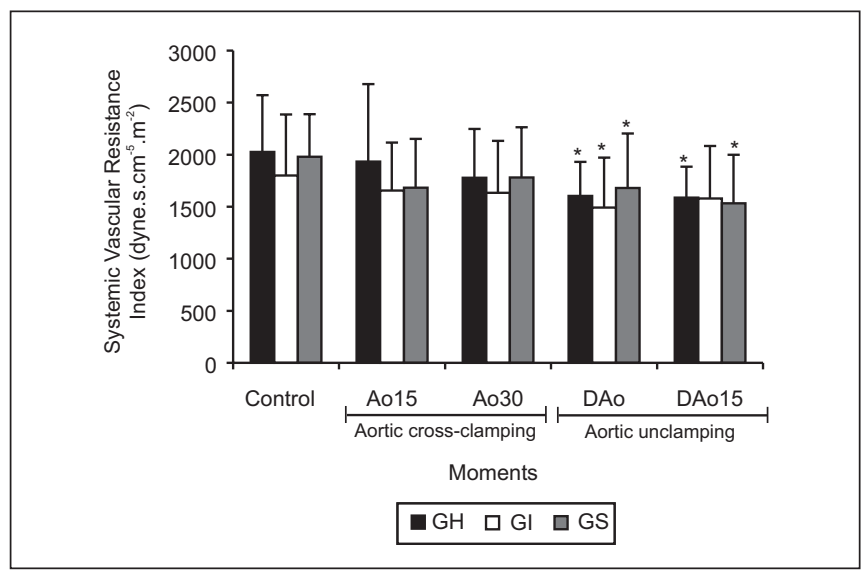

Figure 8 - Systemic Vascular Resistance Index (Mean \pm SD)

${ }^{*} p<0.05$ :

GH: $(C=A 015)>(D A o=D A 015)$

$\mathrm{Gl}: \mathrm{C}>\mathrm{DAO}$

GS: $C>(D A o=D A 015)$

Revista Brasileira de Anestesiologia

Vol. 53, № 4, Julho - Agosto, 2003

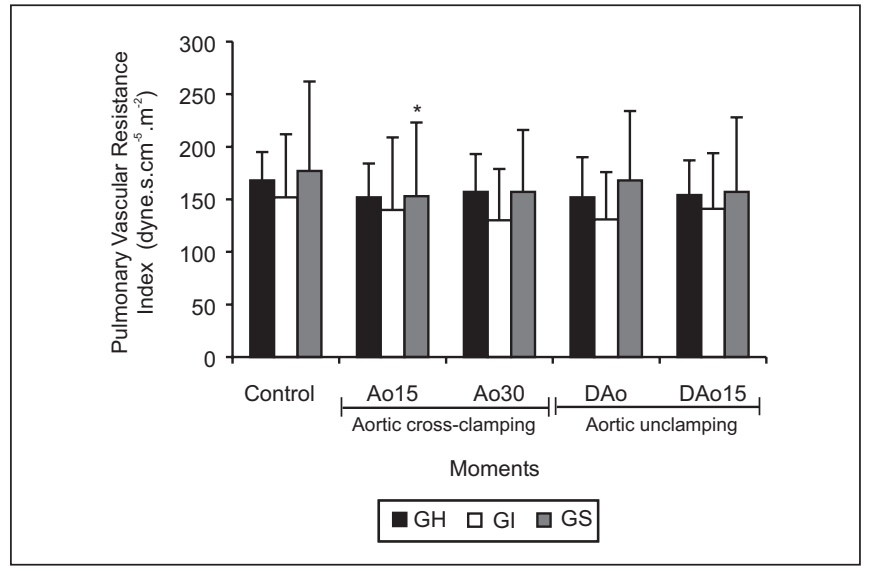

Figure 9 - Pulmonary Vascular Resistance Index (Mean \pm SD) * $p<0.05$ :

GS: C > A015

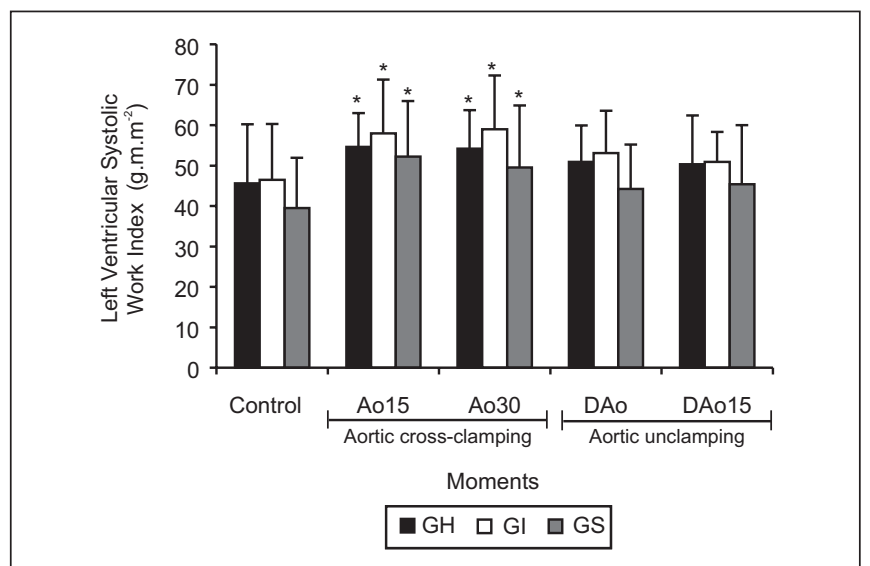

Figure 10 - Left Ventricular Systolic Work Index (Mean \pm SD) ${ }^{*} p<0.05$ :

$\mathrm{GH}: \mathrm{C}<(\mathrm{Ao15}=\mathrm{Ao} 30)$

GI: $\mathrm{C}<($ Ao15 = Ao30 $)>$ DAo15

GS: $\mathrm{C}<(\mathrm{Ao15}=\mathrm{Ao30})>\mathrm{DAo} 15$

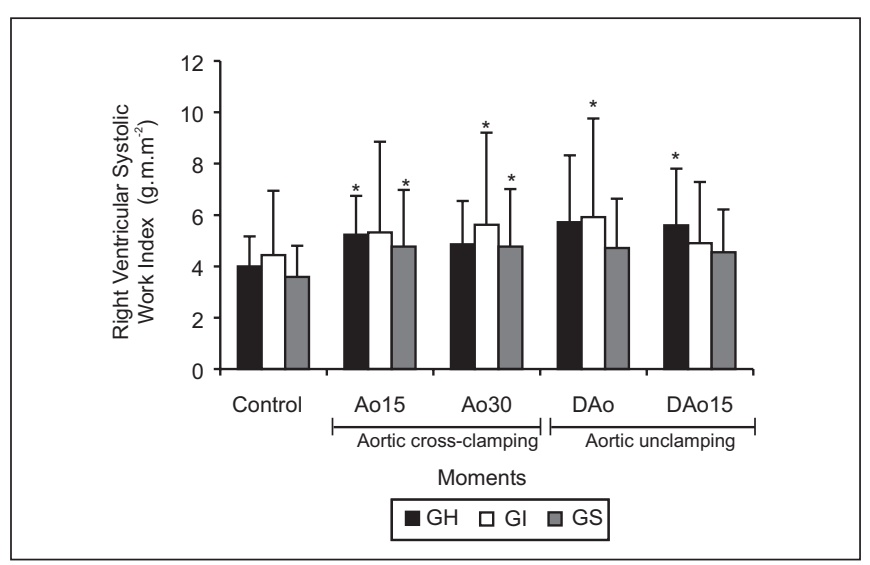

Figure 11 - Right Ventricular Systolic Work Index (Mean \pm SD) ${ }^{*} p<0.05$ :

GH: $C<$ Ao15 < (DAo = DAo15)

GI: $\mathrm{C}<(\mathrm{Ao} 30=\mathrm{DAo})$

GS: $C<(A 015=$ Ao30 $)$ 
Table II - Arterial pH (pHa), Partial Arterial Carbon Dioxide Pressure $\left(\mathrm{PaCO}_{2}\right)$ and Esophageal Temperature $\left(\mathrm{T}_{\mathrm{Esoph}}\right)$, (Mean \pm SD)

\begin{tabular}{|c|c|c|c|c|c|c|}
\hline \multirow[b]{2}{*}{ Attributes } & \multirow[b]{2}{*}{ Groups } & \multicolumn{5}{|c|}{ Moments } \\
\hline & & Control & Ao15 & Ao30 & DAo & DAo15 \\
\hline \multirow[t]{3}{*}{$\mathrm{apH}$} & $\mathrm{GH}$ & $7.35 \pm 0.07$ & $7.33 \pm 0.06$ & $7.31 \pm 0.05^{\star}$ & $7.31 \pm 0.06^{*}$ & $7.29 \pm 0.06^{*}$ \\
\hline & GI & $7.37 \pm 0.06$ & $7.33 \pm 0.05^{*}$ & $7.33 \pm 0.05^{*}$ & $7.31 \pm 0.04^{*}$ & $7.32 \pm 0.05^{*}$ \\
\hline & GS & $7.33 \pm 0.06$ & $7.31 \pm 0.06$ & $7.31 \pm 0.06$ & $7.28 \pm 0.06^{*}$ & $7.28 \pm 0.05^{*}$ \\
\hline \multirow[t]{3}{*}{$\mathrm{PaCO}_{2}(\mathrm{mmHg})$} & $\mathrm{GH}$ & $30 \pm 4$ & $30 \pm 6$ & $32 \pm 6$ & $32 \pm 6$ & $33 \pm 6$ \\
\hline & $\mathrm{GI}$ & $31 \pm 5$ & $32 \pm 5$ & $30 \pm 6$ & $33 \pm 5$ & $32 \pm 3$ \\
\hline & GS & $34 \pm 4$ & $35 \pm 5$ & $35 \pm 4$ & $35 \pm 4$ & $36 \pm 4$ \\
\hline \multirow[t]{3}{*}{$\mathrm{T}_{\text {Esoph }}\left({ }^{\circ} \mathrm{C}\right)$} & $\mathrm{GH}$ & $37.0 \pm 0.8$ & $36.7 \pm 0.8^{*}$ & $36.7 \pm 0.8^{*}$ & $36.6 \pm 0.9^{*}$ & $36.4 \pm 0.8^{*}$ \\
\hline & $\mathrm{GI}$ & $37.2 \pm 0.8$ & $36.9 \pm 0.8^{*}$ & $36.9 \pm 0.9^{*}$ & $36.7 \pm 0.9^{*}$ & $36.6 \pm 0.9^{*}$ \\
\hline & GS & $37.3 \pm 0.6$ & $36.8 \pm 1.0^{*}$ & $36.8 \pm 0.6^{*}$ & $36.7 \pm 0.6^{*}$ & $36.5 \pm 0.7^{*}$ \\
\hline
\end{tabular}

${ }^{*} p<0.05$ as compared to control moment of the same group

\section{DISCUSSION}

Our study has confirmed the presence of hemodynamic changes after aortic cross-clamping and unclamping, even when performed in the lowest aortic levels, such as infrarenal cross-clamping. These changes, which were not significantly different among groups except for heart rate which was significantly lower in the halothane group, were characterized by mild MBP increase (mean of $10 \%$ to $13 \%$ ) which is the most frequent response to aortic cross-clamping, followed by increased filling pressures, such as DBP, PAP and PCWP, and of increased CI, SI, LVSW and RVSW, with significant decrease after aortic unclamping.

Several authors have observed filling pressures increase during aortic cross-clamping ${ }^{9,10}$, while others have not ${ }^{5}$. On the other hand, several authors consider the sudden aortic flow impedance increase with afterload increase ${ }^{11}$, very often followed by $\mathrm{Cl}$ decrease ${ }^{9}$, as the major cause for MBP increase during aortic cross-clamping. In our study, SVRI has not significantly changed in all groups during aortic clamping, while PVRI has significantly decreased in the sevoflurane group during aortic cross-clamping.

So, hemodynamic responses during aortic cross-clamping in this study may be at least partially explained by blood volume redistribution. Quintin et al. (1990) ${ }^{12}$ have observed in men a passive venous withdrawal distally to the aortic crossclamping, with catecholamines (epinephrine and norepinephrine) and angiotensin release. This has determined vasoconstriction both proximal and distal to the cross-clamping with capacitance system decrease and displacement of blood volume proximal to the cross-clamping, with major interference with venous return, that is, in preload, depending on the aortic cross-clamping level be supra or infra-celiac. In the first situation, venous return has always increased because blood volume displacement was toward muscles proximal to the cross-clamping and to lungs and brain. In the infra-celiac, there has been blood volume displacement to splanchnic organs or to other tissues proximal to the 478 cross-clamping. If the splanchnic venous tone is decreased, there will be venous return decrease ${ }^{13}$. As a consequence, blood distribution between splanchnic and non-splanchnic vessels will determine preload changes.

In addition to aortic cross-clamping level, which is the major determinant of hemodynamic changes ${ }^{9}$, other factors may also play a role in aortic cross-clamping responses, such as blood volume or splanchnic vascular tone changes determined by hydration; anesthetic status or drugs action, which may change both blood volume redistribution and venous return; ventricular function; presence and significance of ischemic myocardial disease; and type of aortic disease - aneurysm or occlusive disease ${ }^{2}$.

As to the effects of halogenate anesthetics in patients with no cardiovascular disease submitted to elective surgery, it has been observed that halothane in equipotent doses is more myocardial depressant than isoflurane or sevoflurane ${ }^{14,15}$, while sevoflurane and especially isoflurane have higher vasodilating effect on systemic vascular resistance as compared to halothane ${ }^{16}$ and maintain higher HR as compared to halothane ${ }^{17}$.

In our study, the use of equipotent low doses (0.75 MAC) of each halogenate was certainly decisive for the hemodynamic results obtained. It has to be reminded that HR was the single attribute with significant differences among groups, with lower values in the halothane group as compared to remaining groups during aortic cross-clamping. The comparison of isoflurane and sevoflurane hemodynamic effects in patients with ischemic myocardial disease and hypertension has also showed a similar behavior ${ }^{18}$.

Colson et al. ${ }^{19}(1992)$ have observed in patients submitted to infrarenal aortic reconstruction under anesthesia with $0.64 \%$ halothane or $0.77 \%$ isoflurane that there were no significant hemodynamic differences between groups in the pre-clamping period, such as HR, MBP, PCWP and SVRI. Hemodynamic behavior was also the same during infrarenal aortic cross-clamping with $\mathrm{Cl}$ decrease and SVRI increase, without differences in HR, MBP and PCWP between groups.

Revista Brasileira de Anestesiologia Vol. 53, No 4, Julho - Agosto, 2003 
Results obtained with dogs have also shown similar hemodynamic responses with isoflurane and sevoflurane, with $\mathrm{Cl}$ maintenance at the expenses of HR increase and SVRI decrease. Halothane, however, for not significantly changing HR and for being a stronger myocardial depressant as compared to other halogenate agents, does not maintain $\mathrm{Cl}$ with increased anesthetic depth (2 MAC) ${ }^{20}$.

Seeman-Lodding et al. (1996) ${ }^{5}$, in an experimental model of infrarenal aortic cross-clamping in swine under ketamine and chloralose-based anesthesia, have observed increase in $\operatorname{MBP}(17 \% \pm 4 \%)$ and in SVRI $(27 \% \pm 7 \%)$ during cross-clamping without significant changes in $\mathrm{Cl}, \mathrm{HR}$, DBP, PAP, PCWP and PVRI. In this same study, the authors have introduced a second group receiving $0.7 \%$ isoflurane and then $1.4 \%$ before each cross-clamping, which has determined a dose-dependent decrease in MBP and SVRI, while $\mathrm{HR}$ and $\mathrm{Cl}$ have decreased only with the higher concentration however without major changes in DBP, PAP and PCWP. During aortic cross-clamping, $0.7 \%$ isoflurane has not changed hemodynamic response, but at $1.4 \%$ it has decreased SVRI and MBP. With both isoflurane concentrations there has been MBP decrease in levels below the control group, but during cross-clamping, only the higher concentration has determined MBP levels lower than baseline values.

In our experimental model, mechanisms determining MBP increase during clamping were different from those of the above-described experimental model. Even so, with isoflurane in an intermediate concentration to those used by previous authors, there has been during aortic cross-clamping the same MBP behavior observed in the group receiving $0.7 \%$ isoflurane, that is, there was no impediment for a slight MBP increase.

Using a swine experimental model similar to the above, Sundeman et al. (1996) ${ }^{6}$ have observed that $4.9 \%$ desflurane has not significantly changed hemodynamic responses to infrarenal aortic cross-clamping. However, $9.8 \%$ desflurane has decreased MBP and SVRI during crossclamping.

After aortic unclamping there has been a decrease in most hemodynamic attributes which had increased during aortic cross-clamping, followed by significant decrease in SVRI for all groups, which has favored $\mathrm{Cl}$ and $\mathrm{SI}$ maintenance above baseline values.

Different hemodynamic responses may be seen after aortic unclamping, according to aortic cross-clamping site and its duration, and to patients' hemodynamic status, that is, volume and hemoglobin levels. But there is always a multifactorial SVRI and MBP decrease ${ }^{2}$. Initial response already some minutes after unclamping suggests a reflex or mechanical phenomenon, that is, aortic blood flow increases filling the space created by vasomotor tone decrease, probably as a result of tissue hypoxia, of the build-up of vasodepressant components produced by ischemic tissues, and of myogenic response ${ }^{2,21}$. Blood sequestration in distal veins to the occlusion and increased blood flow in lower limbs are, seemingly, responsible for venous return decrease and, as a consequence, for DBP, $\mathrm{Cl}$ and MBP decrease after aortic unclamping. So, there is the need for an adequate volume replacement to prevent such changes.

In an experimental study with swine under ketamine and chloralose-based anesthesia, it was observed 5 minutes after infrarenal aortic unclamping, that all hemodynamic changes during aortic cross-clamping had returned to baseline levels, with the exception of MBP (+ 10\%) and SVRI (+ $12 \%)$. In this same study, all hemodynamic variables have returned to baseline values after aortic unclamping in the group receiving $4.9 \%$ desflurane, while in the group receiving $9.8 \%$ desflurane there has been MBP decrease $(-7 \%)^{6}$.

In clinical trials with infrarenal aortic cross-clamping followed by unclamping, there has been a decrease in SVRI, MBP and venous return ${ }^{10,19}$. SI and $\mathrm{Cl}$, however, may not change ${ }^{22}$, increase ${ }^{23}$ or decrease ${ }^{24}$, depending on left ventricle filling. Some limitations of human experimental studies with aortic surgeries should be highlighted. So, aortic cross-clamping duration was relatively short and certainly insufficient to determine time-dependent hemodynamic changes. The study was performed in healthy dogs with seemingly normal adaptation ability of the species to different hemodynamic situations, while in men, aortic surgeries are in general performed in elderly patients with some degree of myocardial dysfunction, with occlusive coronary disease, hypertension, chronic obstructive pulmonary disease, renal dysfunction and diabetes mellitus, thus with limited organs reserve. It should also be considered that in this experiment, differently from what happens during human aortic surgeries, bleeding was minor and there has been satisfactory volume replacement, contributing to the maintenance of filling pressures and even attenuating hemodynamic changes caused by aortic unclamping.

Although care has been taken with room temperature, which remained between 24 and $25^{\circ} \mathrm{C}$, and covering dogs with surgical drapes, there has been esophageal temperature decrease in all groups, leading to mild to moderate hypothermia because central temperature of dogs is 38 to $39^{\circ} \mathrm{C}^{25}$. The use of $5 \%$ cold glucose saline solution as an indicator for cardiac output measurement may have also cooperated with the development of hypothermia because each result consisted of three consecutive measurements, thus using $30 \mathrm{ml}$ of the indicator in each moment studied, totaling $150 \mathrm{ml}$ cold glucose saline infusion during the experiment. Nevertheless, hypothermia intensity was not enough to change results obtained in the experiment.

During and after aortic cross-clamping, there has been metabolic acidosis in all groups, probably due to tissue ischemia below the cross-clamping site.

In conclusion, in the conditions used in dogs, halothane, isoflurane and sevoflurane inhalation in equipotent concentrations ( $0.75 \mathrm{MAC})$ has not determined significant changes in major hemodynamic parameters during aortic cross-clamping, characterized by mild filling and mean blood pressures increase. The only exception is heart rate, which is lower with halothane during aortic cross-clamping. The use of low concentrations of halogenate agents has certainly influenced our results. 


\section{REFERÊNCIAS - REFERENCES}

01. Haimovici H - Aneurisma de la Aorta Abdominal, em: Haimovici $\mathrm{H}$ - Cirugia Vascular - Principios y Tecnicas. $2^{\mathrm{a}} \mathrm{Ed}$, Barcelona: Salvat Editores, 1986;656-707.

02. Gelman S - The pathophysiology of aortic cross-clamping and unclamping. Anesthesiology, 1995;82:1026-1060

03. Jamieson WRE, Janusz MT, Miyagishima RT et al - Influence of ischemic heart disease on early and late mortality after surgery for peripheral occlusive vascular disease. Circulation, 1982;66: (Suppl I):92-97.

04. Colson P, Capdevilla X, Balert H et al - Effects of halothane and isoflurane on transient renal dysfunction associated with infrarenal aortic cross-clamping. J Cardioth Vasc Anesth, 1992;6:295-298.

05. Seeman-Lodding H, Biber B, Martner J et al - Cardiovascular responses to experimental infra-renal aortic cross-clamping. Modulating effects of isoflurane, sodium nitroprussiate and milrinone. Acta Anaesthesiol Scand, 1996;40:408-415.

06. Sundeman H, Biber B, Heniksson BA, et al - Effects of desflurane on systemic, preportal and renal circulatory responses to infra-renal aortic cross-clamping in the pig. Acta Anaesthesiol Scand, 1996;40:876-882.

07. Kazama T, Ikeda K - Comparison of MAC and the rate of rise of alveolar concentration of sevoflurane with halothane and isoflurane in the dog. Anesthesiology, 1988;68:435-437.

08. Morrison DF - Multivariate Statistical Methods. New York, Mc Graw Hill, 1967;338.

09. Roisen MF, Beaupre PN, Alpert RA et al - Monitoring with two-dimensional transesophageal echocardiography: Comparison of myocardial function in patients undergoing supra-celiac, suprarenal-infraceliac, or infrarenal aortic occlusion. J Vasc Surg, 1984;1:300-305.

10. Harpole DH, Clements FM, Quill T et al - Right and left ventricular performance during and after abdominal aortic aneurysm repair. Ann Surg, 1989;209:356-362.

11. Kien ND, White DA, Reitan JA et al - The influence of adenosine triphosphate on left ventricular function and blood flow distribution during aortic crossclamping in dogs. J Cardiothor Vasc Anesth, 1987;1:114-122.

12. Quintin L, Bonnet F, Macquin I et al - Aortic surgery: effect of clonidine on intra-operative catecholaminergic and circulatory stability. Acta Anaesthesiol Scand, 1990;34:132-137.

13. Vandermeer TJ, Maini BS, Hendershott TH et al - Evaluation of right ventricular function during aortic operations. Arch Surg, 1993;128:582-585

14. Eger II EI - New inhaled anesthetics. Anesthesiology, 1994;80: 906-922.

15. Heerdt PM, Pleimann BE - The dose-dependent effects of halothane on right ventricular contraction pattern and regional inotropy in swine. Anesth Analg, 1996;82:1152-1158.

16. Heerdt PM, Gandhi CD, Dickstein ML - Disparity of isoflurane effects on left and right ventricular after load and hydraulic power generation in swine. Anesth Analg, 1998;87:511-521.

17. Malan TP, Dinardo JA, Isner RJ et al - Cardiovascular effects of sevoflurane compared with those of isoflurane in volunteers. Anesthesiology, 1995;83:918-928.

18. Rooke AG, Ebert T, Muzi M et al - The hemodynamic and renal effects of sevoflurane and isoflurane in patients with coronary artery disease and chronic hypertension. Anesth Analg, 1996;82: 1159-1165.

19. Mutoh T, Nishimura R, Kim HY et al - Cardiopulmonary effects of sevoflurane, compared with halothane, enflurane, and isoflurane in dogs. Am J Vet Res, 1997;58:885-890.
20. Colson P, Ribsteien J, Séguin JR et al - Mechanism of renal hemodynamic impairment during infrarenal aortic cross-clamping. Anesth Analg, 1992;75:18-23.

21. Nielsen VG - Xanthyne oxidoreductase release after descending thoracic aorta occlusion and reperfusion in rabbits. J Thorac Cardiovasc Surg, 1994;107:1222-1227.

22. Reiz S, Peter T, Rais O - Hemodynamic effects of infra-renal and common iliac artery desclamping in man. An approach to optimal volume loading. Acta Anaesthesiol Scand, 1979;23: 579-586.

23. Colson P, Capdevilla X, Cuchet D et al - Does choice of the anesthetic influence renal function during infrarenal aortic surgery? Anesth Analg, 1992;74:481-485.

24. Reis JMV, Braz JRC, Pereira SM et al - Comportamento hemodinâmico de pacientes portadores de aneurisma da aorta abdominal e de síndrome de Leriche durante enxerto aorto bifemoral. Rev Bras Anestesiol, 1994;44:(Supl18):CBA155.

25. Massone F - Anestesiologia Veterinária. $3^{\mathrm{a}} \mathrm{Ed}$, Rio de Janeiro, Guanabara Koogan, 1999;225.

\section{RESUMEN}

Bisinotto FMB, Braz JRC - Efectos del Halotano, Isoflurano y Sevoflurano en las Respuestas Cardiovasculares al Pinzamiento Aórtico Infra-Renal. Estudio Experimental en Perros

JUSTIFICATIVA Y OBJETIVOS: EI pinzamiento infra-renal de la aorta abdominal puede producir alteraciones hemodinámicas. El objetivo del estudio fue evaluar los efectos del halotano, isoflurano y sevoflurano sobre la función cardiovascular, en perros sometidos al pinzamiento aórtico infra-renal.

MÉTODO: El estudio aleatorio fue realizado en 30 perros, distribuidos en tres grupos, de acuerdo con el anestésico halogenado utilizado durante la anestesia, en concentraciones equipotentes de 0,75 CAM: GH $(n=10)$ - halotano a 0,67\%; GI $(n=10)$ - isoflurano a $0,96 \%$; y $G S(n=10)$ - sevoflurano a $1,8 \%$. En todos los animales fue realizada ligadura infra-renal de la aorta, por período de $30 \mathrm{~min}$. Los atributos hemodinámicos fueron estudiados en los momentos: C (Control), Ao15 y Ao 30, respectivamente después de15 y $30 \mathrm{~min}$ del pinzamiento aórtico, y DAo y DAo15, respectivamente, inmediatamente y después de15 min del despinzamiento aórtico.

RESULTADOS: Durante el pinzamiento aórtico hubo, en todos los grupos, aumento de las presiones arterial media y del átrio derecho, y de los índices cardíaco, sistólico y de trabajo sistólico de los ventrículos derecho e izquierdo. La presión de la arteria pulmonar aumentó en GI y GS y la presión pulmonar ocluida en GH y GI. Después del despinzamiento aórtico, hubo normalización de los atributos que se habían elevado, con excepción de los índices cardíaco y sistólico, que continuaron elevados, acompañados de diminución del índice de resistencia vascular sistémica. No hubo diferencia significante entre los grupos en relación a los atributos estudiados, con excepción de la frecuencia cardíaca que fue siempre menor en $\mathrm{GH}$, en relación a los demás grupos, durante el pinzamiento y despinzamiento aórtico.

CONCLUSIONES: En el perro, en las condiciones experimentales empleadas, la inhalación del halotano, isoflurano y sevoflurano en concentraciones equipotentes (0,75 CAM) no atenúa las respuestas cardiovasculares al pinzamiento aórtico infra-renal. 